\title{
HOLOMORPHIC HOROSPHERICAL TRANSFORM ON NON-COMPACTLY CAUSAL SPACES
}

\author{
SIMON GINDIKIN, BERNHARD KRÖTZ AND GESTUR ÓLAFSSON
}

\begin{abstract}
We develop integral geometry for non-compactly causal symmetric spaces. We define a complex horospherical transform and, for some cases, identify it with a Cauchy type integral.
\end{abstract}

\section{INTRODUCTION}

Within the class of pseudo Riemannian symmetric spaces there are causal symmetric spaces $Y=G / H$ with an invariant generalized conformal structure defined by a field of (linear equivalent ) convex cones [14]. There are 2 types of causal structures which correspond to 2 types of such spaces: compactly causal (CC) and noncompactly causal spaces (NCC) [14. In both cases there exists $G$-invariant Stein tubes $D=D(Y)$ in the Stein symmetric space $Y_{\mathbb{C}}=G_{\mathbb{C}} / H_{\mathbb{C}}$ which have $Y$ as Shilov boundary. There are some similarities but also some substantial differences with regard to analysis on these tubes. The most important fact is that one can realize different series of representations in Hilbert spaces of holomorphic functions on $D$ : holomorphic discrete series on $Y$ for the CC-case in contrast to a multiplicity one subspace of the most continuous spectrum in the NCC-case.

In [9] we developed integral geometry for $D$ in the CC-case. If to consider the usual (real) horospherical transform on $Y$, then holomorphic discrete series lie in its kernel. So we considered a complex version of such a transform - horospherical Cauchy transform using a kernel of Cauchy type with singularities on complex horospheres (on $Y_{\mathbb{C}}$ ) which do not intersect $Y$. As a result we constructed a dual domain $\Xi_{+}$in the manifold $\Xi$ of complex horospheres on $Y_{\mathbb{C}}$ and our horospherical transform is an intertwining operator from holomorphic functions on $D$ to holomorphic functions on $\Xi_{+}$which admits an explicit inversion.

In this paper we try to realize a similar program for NCC-spaces. The situation here is different. The real horospherical transform on $Y$ is injective on the most continuous spectrum but it does not make a difference between different multiplicities of this spectrum. Thus we want find such a modification of this transform which separates one multiplicity of most continuous spectrum. It turns out that such a construction exists and again it has a complex

Key words and phrases. Radon transform, horospheres, Hardy spaces.

SG was supported in part by NSF-grant DMS-0070816.

BK was supported by the RiP-program in Oberwolfach, and a Heisenberg fellowship of the DFG.

GÓ was supported by the RiP-program in Oberwolfach, NSF grants DMS-0139783 and DMS-0402068. 
nature. An essential difference of the NCC-case compared to the CC-case is that there are fewer complex horospheres for $Y_{\mathbb{C}}$ which do not intersect $Y$ : they are parameterized by some $C R$-manifold of complex dimension equal to the real rank of $Y$. Anyway it is sufficient to have an appropriate construction.

Our basic construction differs from the one in the CC-case. We use the fact that $D$ in the NCC-case contains the Riemannian symmetric space $X$ for the group $G$. We remark that the the (real) horospherical transform on holomorphic functions on $D$, restricted on $X$, admit holomorphic extension as $C R$-function on the $C R$ manifold $\Xi_{+}$, parametrizing complex horospheres which do not intersect $Y$. Let us point out that integrals on all real horospheres in the intersection of such a complex horosphere with $D$ coincide. We call such a transform holomorphic horospherical transform. Of course we can invert it using the inversion of horospherical transform on $X$. In some cases (the most interesting case of spaces of Caley type) we can rewrite this transform as some Cauchy type integral on $Y$. We call such form of the horospherical transform the horospherical Cauchy transform similarly to the CC-case.

The paper is concluded with a geometric definition of the most continuous Hardy space introduced in 9 .

\section{Horospheres on NCC SYMMETRIC SPACES}

In [9] we associated to every NCC symmetric space $Y=G / H$ a $G$-Stein manifold $D$ with the following properties:

(1) The complex manifold $D$ has a natural $G$-realization in the complexification $Y_{\mathbb{C}}$ of $Y$

(2) The symmetric space $Y=G / H$ is $G$-isomorphic to the distinguished (Shilov) boundary of $D$.

The objective of this section is to study the space $\Xi=G_{\mathbb{C}} / M_{\mathbb{C}} N_{\mathbb{C}}$ of horospheres in $Y_{\mathbb{C}}$ in relation to $D$. In particular we will introduce a natural $G$-invariant $C R$-manifold $\Xi_{+} \subset \Xi$ whose elements have the properties that they do non intersect the real space $Y$, i.e. have no real points.

1.1. Notation. We informally recall the notion of an NCC space. For that let $G / H$ be a semisimple symmetric space. We assume that the group $G$ is connected, simple and that $H$ is the full fixed point group of the underlying involution $\tau: G \rightarrow G$, i.e., $H=G^{\tau}$. We write $y_{o}=H$ for the base point of $Y$ and identify the tangent space $T_{y_{o}} Y$ with the -1-eigenspace $\mathfrak{q}$ of $d \tau(\mathbf{1})$. Then $Y$ is called non-compactly causal $(N C C)$ if there exists an hyperbolic element $0 \neq Z_{H} \in \mathfrak{q}=T_{y_{o}} Y$ such that $Z_{H}$ generates a proper $\operatorname{Ad}(H)$-invariant open convex cone (cf. [14]). We can assume that $Z_{H}$ is $H \cap K$-invariant, where $K$ is a $\tau$-stable maximal compact subgroup of $G$. Let us also mention that there there are the compactly causal spaces (CC) which are dual to the NCC-spaces [14.

Let us fix a maximal abelian subspace $\mathfrak{a} \subset \mathfrak{q}$ which contains $Z_{H}$. We recall that $\mathfrak{a}$ is hyperbolic and that we can choose $Z_{H}$ in such a way that $\operatorname{ad}\left(Z_{H}\right)$ defines a 3 -grading with 
$\pi / 2$ the maximal eigenvalue. Define $\Omega_{H} \subseteq \mathfrak{a}$ by

$$
\Omega_{H}=\operatorname{int}\left\{\text { convex hull of } \mathcal{W}\left(Z_{H}\right)\right\} \text {. }
$$

Here $\mathcal{W}$, as usually, denotes the Weyl group of the restricted root system $\Sigma=\Sigma(\mathfrak{g}, \mathfrak{a})$ with $\mathfrak{g}$ the Lie algebra of $G$. Observe that $\bar{\Omega}_{H}$ is a compact convex subset of $\mathfrak{a}$ with extreme points $\mathcal{W}\left(Z_{H}\right)$.

Attached to $Y$ and $\Omega_{H}$ comes a Stein manifold $D$ which we will now describe. Denote by $G_{\mathbb{C}}$ the universal complexification of $G$. It is convenient and no big loss of generality to require that $G_{\mathbb{C}}$ is simply connected with $G \subset G_{\mathbb{C}}$. Under these assumptions $\tau$ extends to a holomorphic involution of $G_{\mathbb{C}}$ and with the corresponding fixed point group $H_{\mathbb{C}}$ we obtain a totally real embedding

$$
Y \hookrightarrow Y_{\mathbb{C}}=G_{\mathbb{C}} / H_{\mathbb{C}}
$$

of $Y$ in the Stein symmetric space $Y_{\mathbb{C}}$.

According to Berger there exists a Cartan involution $\theta: G \rightarrow G$ which commutes with $\tau$. In addition we may and will assume that $\mathfrak{a}$ is included in the -1-eigenspace $\mathfrak{p}$ of $d \theta(\mathbf{1})$. Write $K$ for the compact group of $\theta$-fixed elements and $X=G / K$ for the corresponding Riemann symmetric space. As before we obtain a totally real embedding

$$
X \hookrightarrow X_{\mathbb{C}}=G_{\mathbb{C}} / K_{\mathbb{C}}
$$

Recall that $H_{\mathbb{C}}$ and $K_{\mathbb{C}}$ are conjugate, i.e. with $z_{H}=\exp \left(i Z_{H}\right)$ we have cf. [9, 20]:

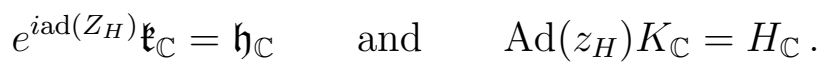

Hence $X_{\mathbb{C}}$ and $Y_{\mathbb{C}}$ are canonically $G_{\mathbb{C} \text {-isomorphic via the map }}$

$$
X_{\mathbb{C}} \ni g K_{\mathbb{C}} \mapsto g z_{H}^{-1} H_{\mathbb{C}} \in Y_{\mathbb{C}} .
$$

In the sequel we identify $X_{\mathbb{C}}$ with $Y_{\mathbb{C}}$.

We write $x_{o}=K_{\mathbb{C}} \in X_{\mathbb{C}}$ for the base point in $X_{\mathbb{C}}$ and set

$$
D=G \exp \left(i \Omega_{H}\right) \cdot x_{o} .
$$

Note that $D$ was denoted by $\Xi_{H}$ in our previous article 9 . According to 9 it is known that $D$ is an open $G$-invariant Stein neighborhood of $X$ in $X_{\mathbb{C}}=Y_{\mathbb{C}}$. Moreover, the map $Y=G / H \ni g H \mapsto g z_{H} \cdot x_{o} \in X_{\mathbb{C}}$ identifies $Y$ with the distinguished boundary $\partial_{d} D$ of $D$ (see 9], Section 1, for more details).

In summary, the symmetric Stein manifold $X_{\mathbb{C}}=Y_{\mathbb{C}}$ admits 2 real forms $X$ and $Y$ and a Stein neighborhood $D$ of $X$ with $Y$ as its Shilov boundary.

1.2. Complex horospheres. In this section we introduce the $G$-space of horospheres in the complex manifold $X_{\mathbb{C}}$. This was done for CC-spaces in [10].

We begin with some general remarks on convexity which we will use frequently. Let $G=N A K$ be an Iwasawa decomposition of $G$ and $N_{\mathbb{C}} A_{\mathbb{C}} K_{\mathbb{C}} \subsetneq G_{\mathbb{C}}$ its Zariski-open complexification. In particular, $N_{\mathbb{C}} A_{\mathbb{C}} \cdot x_{o}$ is a Zariski-open subset in the affine variety $X_{\mathbb{C}}$. Define the finite 2-group $F=A_{\mathbb{C}} \cap K_{\mathbb{C}}$ and note that there are well defined holomorphic maps 


$$
n: N_{\mathbb{C}} A_{\mathbb{C}} \cdot x_{o} \rightarrow N_{\mathbb{C}}, \quad a: N_{\mathbb{C}} A_{\mathbb{C}} \cdot x_{o} \rightarrow A_{\mathbb{C}} / F
$$

such that $z=n(z) a(z) \cdot x_{o}$ for all $z \in N_{\mathbb{C}} A_{\mathbb{C}} \cdot x_{o}$. Now, the fact that $D$ is contractible and $D \subset N_{\mathbb{C}} A_{\mathbb{C}} \cdot x_{o}$, implies that $\left.a\right|_{D}$ admits a well defined holomorphic logarithm

$$
\log a: D \rightarrow \mathfrak{a}_{\mathbb{C}}
$$

For $Z \in \Omega_{H}$, the complex convexity theorem (cf. [8, 16]) then implies that

$$
\operatorname{Im} \log a\left(G \exp (i Z) \cdot x_{o}\right)=\operatorname{conv}(\mathcal{W} \cdot Z)
$$

where $\operatorname{conv}(\cdot)$ denotes the convex hull of $(\cdot)$.

Submanifolds of $X_{\mathbb{C}}$ of the type

$$
g N_{\mathbb{C}} \cdot x_{0} \quad\left(g \in G_{\mathbb{C}}\right)
$$

will be refered as horospheres. We denote by $\operatorname{Hor}\left(X_{\mathbb{C}}\right)$ the set of all horospheres on $X_{\mathbb{C}}$ and note that $\operatorname{Hor}\left(X_{\mathbb{C}}\right)$ has a natural $G$-structure $\left(g, h N_{\mathbb{C}} \cdot x_{o}\right) \mapsto g h N_{\mathbb{C}} \cdot x_{o}$.

To understand the space horospheres and the related harmonic analysis it is useful to bring them in the context of a double fibration. Set $M=Z_{K}(A) \subset M_{\mathbb{C}}=Z_{K_{\mathbb{C}}}(A)$, define

$$
\Xi=G_{\mathbb{C}} / M_{\mathbb{C}} N_{\mathbb{C}}
$$

and consider:

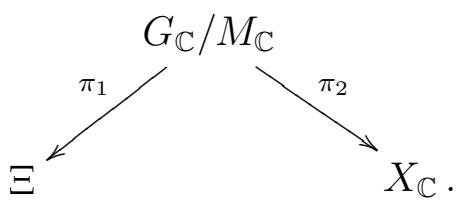

Then horospheres in $X_{\mathbb{C}}$ are exactly the subsets of $X_{\mathbb{C}}$ of the form

$$
E(\xi)=\pi_{2}\left(\pi_{1}^{-1}(\xi)\right) \quad(\xi \in \Xi) .
$$

If $\xi_{o}=M_{\mathbb{C}} N_{\mathbb{C}} \in \Xi$ denotes the base point and $\xi=g \cdot \xi_{o} \in \Xi$ then, using that $M_{\mathbb{C}} \subset H_{\mathbb{C}}$, we have:

$$
E(\xi)=g M_{\mathbb{C}} N_{\mathbb{C}} \cdot x_{o}=g N_{\mathbb{C}} \cdot x_{o} \subset X_{\mathbb{C}} .
$$

Similarly, for $z \in X_{\mathbb{C}}$ we set

$$
S(z)=\pi_{1}\left(\pi_{2}^{-1}(z)\right) .
$$

If $z=g \cdot x_{o}$ for $g \in G_{\mathbb{C}}$, then $S(z)=g K_{\mathbb{C}} \cdot \xi_{o}$. Moreover, for $z \in X_{\mathbb{C}}$ and $\xi \in \Xi$ one has the incidence relations

$$
z \in E(\xi) \Longleftrightarrow \pi_{1}^{-1}(\xi) \cap \pi_{2}^{-1}(z) \neq \emptyset \Longleftrightarrow \xi \in S(z) .
$$

Proposition 1.1. The map

$$
\Xi \rightarrow \operatorname{Hor}\left(X_{\mathbb{C}}\right), \quad \xi \mapsto E(\xi)
$$

is a $G_{\mathbb{C}}$-equivariant bijection. 


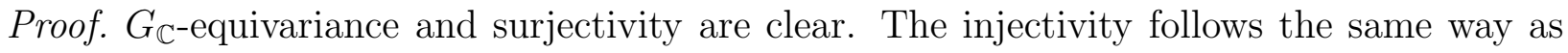
in the proof of Proposition 2.1 in [10] by replacing $H_{\mathbb{C}}$ by $K_{\mathbb{C}}$.

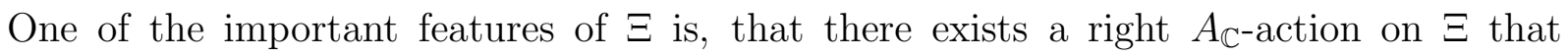
commutes with the left $G_{\mathbb{C}^{-}}$action. For $\xi=g \cdot \xi_{0}$ and $a \in A_{\mathbb{C}}$ we set

$$
\xi \cdot a=g a \cdot \xi_{0}
$$

Since $A_{\mathbb{C}}$ normalizes $M_{\mathbb{C}} N_{\mathbb{C}}$ it is clear that (1.8) is well defined. From the definition it is also

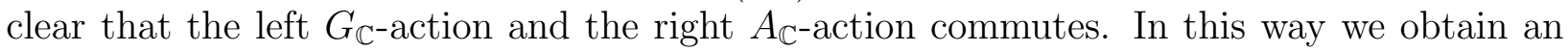
action of $G_{\mathbb{C}} \times A_{\mathbb{C}}$ on $\Xi$ by

$$
\left(G_{\mathbb{C}} \times A_{\mathbb{C}}\right) \times \Xi \rightarrow \Xi, \quad((g, a), \xi) \mapsto g \cdot \xi \cdot a .
$$

We conclude this subsection with an alternative characterization of horospheres as level sets of holomorphic functions. For that let $\left\{\omega_{1}, \ldots, \omega_{n}\right\} \subset \mathfrak{a}^{*}$ be the set of fundamental $K$-spherical lowest weights. For each $1 \leq j \leq n$ we write $\left(\pi_{j}, V_{j}\right)$ for the corresponding finite dimensional representation of $G$ with lowest weight $\omega_{j}$. We extend this representation to a holomorphic representation of $G_{\mathbb{C}}$ which we denote by the same symbol. Endow $V_{j}$ with a complex bilinear pairing $\langle$,$\rangle such that \left\langle\pi_{j}(g) v, w\right\rangle=\left\langle v, \pi_{j}\left(\theta(g)^{-1}\right) w\right\rangle$ for all $v, w \in V_{j}$ and $g \in G_{\mathbb{C}}$. Such a form $\langle$,$\rangle exists as \pi \circ \theta$ is isomorphic to the representation contragradient to $\pi_{j}$. We write $v_{j} \in V_{j}$ for a lowest weight vector and $\eta_{j} \in V_{j}$ for a $K_{\mathbb{C}}$-fixed vector subject to the normalization $\left\langle\eta_{j}, v_{j}\right\rangle=1$. Finally, define holomorphic functions on $f_{j}: G_{\mathbb{C}} \rightarrow \mathbb{C}$ by

$$
f_{j}(g)=\left\langle\pi_{j}(g) \eta_{j}, v_{j}\right\rangle \quad\left(g \in G_{\mathbb{C}}\right)
$$

Note, that we have

$$
f_{j}(n a k)=a^{\omega_{j}}
$$

for all $n \in N_{\mathbb{C}}, k \in K_{\mathbb{C}}$ and $a \in A_{\mathbb{C}}$. Here, as elsewhere in this article, we use the notation $a^{\mu}=e^{\mu(X)}$ if $a=\exp X \in A_{\mathbb{C}}$. We recall that

$$
G_{\mathbb{C}} \backslash N_{\mathbb{C}} A_{\mathbb{C}} K_{\mathbb{C}}=\left\{g \in G_{\mathbb{C}} \mid \prod_{j=1}^{n} f_{j}(g)=0\right\} .
$$

(see [18, Th. 2.5 for a proof in a more general context).

Lemma 1.2. $N_{\mathbb{C}} K_{\mathbb{C}}=\left\{g \in G_{\mathbb{C}} \mid f_{j}(g)=1 \quad\right.$ for all $\left.\quad 1 \leq j \leq n\right\}$.

Proof. This follows from (1.10) and (1.11).

We will often view $f_{j}$, or more generally it left translates, as a function on $X_{\mathbb{C}}$. We will also, without further comments, view the function $g \mapsto f_{j}\left(g^{-1} z\right), z \in X_{\mathbb{C}}$ as a function on $\Xi$. With that in mind we have: 
Lemma 1.3. Let $\xi \in \Xi$ and $x \in X_{\mathbb{C}}$. Then

$$
E(\xi)=\left\{z \in X_{\mathbb{C}} \mid f_{j}\left(\xi^{-1} z\right)=1 \quad \text { for all } \quad 1 \leq j \leq n\right\}
$$

and

$$
S(x)=\left\{\varrho \in \Xi \mid f_{j}\left(\varrho^{-1} x\right)=1 \quad \text { for all } \quad 1 \leq j \leq n\right\}
$$

Proof. Notice that $E\left(g \cdot \xi_{o}\right)=g E\left(\xi_{o}\right)$ and $S\left(g \cdot x_{o}\right)=g S\left(x_{o}\right)$. We can therefore assume that $\xi=\xi_{o}$ and $x=x_{0}$. Now, the claim is a reformulation of Lemma 1.2.

1.3. Some $G$-submanifolds of $\Xi$. We define the $G$-space of real horospheres in $X$ as

$$
\Xi_{\mathbb{R}}=G / M N \text {. }
$$

Then $\Xi_{\mathbb{R}} \subset \Xi=G_{\mathbb{C}} / M_{\mathbb{C}} N_{\mathbb{C}}$ is obviously a totally real $G$-submanifold of $\Xi$ and the right $A$-action leaves $\Xi_{\mathbb{R}}$ invariant.

Let $T=\exp (i \mathfrak{a}) \subset G_{\mathbb{C}}$ and note that $A_{\mathbb{C}}=A \times T$; note that $F=K \cap T$. We contrast $\Xi_{\mathbb{R}}$

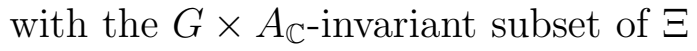

$$
\Xi_{0}=G \cdot \xi_{0} \cdot A_{\mathbb{C}}=G A_{\mathbb{C}} \cdot \xi_{0} .
$$

Proposition 1.4. The following assertion hold:

(1) The map

$$
K / M \times_{F} A_{\mathbb{C}} \rightarrow \Xi_{0}, \quad[k M, a] \mapsto k a \cdot \xi_{o}
$$

is a real analytic isomorphism.

(2) The map

$$
\Xi_{\mathbb{R}} \times_{F} T \rightarrow \Xi_{0}, \quad[g M N, t] \mapsto g t \cdot \xi_{o}
$$

is a $G$-equivariant real analytic diffeomorphism.

Proof. (i) follows from the fact that $G=K A N$ and $N A_{\mathbb{C}} \subset A_{\mathbb{C}} N_{\mathbb{C}}$. Finally (ii), is a consequence of (i).

Note that (1.13) describes a natural $C R$-structure on $\Xi_{0}$ of $C R$-dimension $\operatorname{dim} A$ and $C R$-codimension $\operatorname{dim} K / M$.

Define a tube domain in $A_{\mathbb{C}}$ by

$$
T\left(\Omega_{H}\right)=\exp \left(\mathfrak{a}+i \Omega_{H}\right)=A \exp \left(i \Omega_{H}\right) \simeq \mathfrak{a}+i \Omega_{H}
$$

and set

$$
\Xi_{+}=G \exp \left(i \Omega_{H}\right) \cdot \xi_{o}=K T\left(\Omega_{H}\right) \cdot \xi_{o} .
$$

Then $\Xi_{+}$is a real-analytic, $G$-invariant open submanifold of $\Xi_{0}$. In particular $\Xi_{+}$is a $C R$ manifold. The coordinate decomposition of $\Xi_{0}$ slightly simplifies for $\Xi_{+}$.

Proposition 1.5. For $\Xi_{+}$the following assertions hold: 
(1) The map

$$
K / M \times T\left(\Omega_{H}\right) \rightarrow \Xi_{+}, \quad(k M, a) \mapsto k a \cdot \xi_{o}
$$

is a real analytic isomorphism.

(2) The map

$$
\Xi_{\mathbb{R}} \times \Omega_{H} \rightarrow \Xi_{+}, \quad(g M N, Z) \mapsto g \exp (i Z) \cdot \xi_{o}
$$

is a $G$-equivariant real analytic diffeomorphism.

We conclude this section with a remark on the structure of $\Xi_{+}$.

Remark 1.6. It is immediate from the complex convexity theorem (1.3) that

$$
\Xi_{+}=\left\{\xi \in \Xi_{0} \mid E(\xi) \cap D \neq \emptyset\right\}_{0}
$$

with $\{\cdot\}_{0}$ the connected component of $\Xi_{0}$ containing $\Xi_{\mathbb{R}}$. In other words, $\Xi_{+}$is the connected component of $\Xi_{0}$ which contains $\Xi_{\mathbb{R}}$ and has the property that the corresponding horospheres do intersect $D$.

Remark 1.7. (Shilov boundary of $\Xi_{+}$) The map

$$
\Xi_{\mathbb{R}} \rightarrow \partial \Xi_{+}, \quad g M N \mapsto g z_{H} \cdot \xi_{o}
$$

identifies $\Xi_{\mathbb{R}}$ as the Shilov boundary $\partial_{S} \Xi_{+}$of $\Xi_{+}$. In this sense $\Xi_{\mathbb{R}}$ parametrizes the real horospheres on $Y$ (see also Lemma 1.8 below).

1.4. Horospheres without real points. The aim of this subsection is to show that horospheres corresponding to $\Xi_{+}$do not contain real points, i.e., are disjoint form $Y$.

Recall from Subsection 1.1 that we identify $Y=G / H$ with the (Shilov) boundary orbit $G \cdot y_{o} \subset X_{\mathbb{C}}$ of $y_{o}=z_{H} \cdot x_{o}$ in $G_{\mathbb{C}} / K_{\mathbb{C}}$. Define the parameter set of horospheres without real points by

$$
\Xi_{\mathrm{nr}}=\{\xi \in \Xi \mid E(\xi) \cap Y=\emptyset\} .
$$

The following statement should be comparedto the complex convexity Theorem (1.3); it means that convexity breaks down at the extreme points of $\Omega_{H}$.

Lemma 1.8. Let $\mathcal{U}=\bigcup_{w \in \mathcal{W}} N A w H$. Then $\mathcal{U} \cdot y_{o}$ is open and dense in $G \cdot y_{o}$ and

$$
G \cdot y_{o} \cap N_{\mathbb{C}} A_{\mathbb{C}} \cdot x_{o}=\mathcal{U} \cdot y_{o}=N A \mathcal{W} z_{H} \cdot x_{o} .
$$

Proof. It is a special case of a Theorem by Rossmann-Matsuki (cf. 17]) that $\mathcal{U}$ is dense in $G$. Hence $\mathcal{U} \cdot y_{o}$ is dense in $G \cdot y_{o}$. As $z_{H}^{-1} H_{\mathbb{C}} z_{H}=K_{\mathbb{C}}$ (cf. (1.2), it follows that $\mathcal{U} \cdot y_{o}=N A \mathcal{W} z_{H} \cdot x_{o}$. It remains to show the inclusion " $\supseteq$ " for the first asserted equality. But this follows from (1.11).

We can now prove the main result of this subsection.

Theorem 1.9. $\Xi_{+} \subseteq \Xi_{\mathrm{nr}}$. 
Proof. We argue by contradiction. Note that $E(\xi) \cap Y \neq \emptyset$ for some $\xi \in \Xi_{+}$means that there exist $Z \in \Omega_{H}$ such that

$$
G z_{H} \cdot x_{o} \cap \exp (i Y) N_{\mathbb{C}} \cdot x_{o} \neq \emptyset .
$$

Now $\exp (i Y) N_{\mathbb{C}} \cdot x_{o}=N_{\mathbb{C}} \exp (i Y) \cdot x_{o} \subset N_{\mathbb{C}} \exp \left(i \Omega_{H}\right) \cdot x_{o}$ and the assertion follows from Lemma 1.8.

1.5. Real forms of $E(\xi)$ and $S(z)$. In this last part of this section we introduce certain $G$-invariant real forms of the complex manifolds $E(\xi)$ and $S(z)$.

We begin with the horospheres. For $\xi=g a \cdot \xi_{o} \in \Xi_{+}$, with $g \in G$ and $a \in \exp \left(i \Omega_{H}\right)$, define

$$
E_{\mathbb{R}}(\xi)=g N a \cdot x_{o} \subset E(\xi) .
$$

Then $E_{\mathbb{R}}(\xi)$ is well defined, $G$-invariant and a totally real submanifold of $E(\xi)$. Further, the assignment $\Xi_{+} \ni \xi \mapsto E_{\mathbb{R}}(\xi)$ is $G$-equivariant.

Next we consider $S(z) \simeq K_{\mathbb{C}} / M_{\mathbb{C}}$. Because of the relation $K_{\mathbb{C}}=z_{H}^{-1} H_{\mathbb{C}} z_{H}$ there are two natural real forms. Accordingly we define for $z=g a \cdot x_{o} \in D$ :

$$
S_{\mathbb{R}}^{K}(z)=g a K \cdot \xi_{o} \quad \text { and } \quad S_{\mathbb{R}}^{H}(z)=g a z_{H}^{-1} H z_{H} \cdot \xi_{o} .
$$

Obviously $S_{\mathbb{R}}^{K}(z)$ and $S_{\mathbb{R}}^{H}(z)$ are $G$-invariant totally real submanifold of $S(z)=g a K_{\mathbb{C}} \cdot x_{o}$ and the maps $D \ni z \mapsto S_{\mathbb{R}}^{K}(z)$ and $D \ni z \mapsto S_{\mathbb{R}}^{H}(z)$ are $G$-equivariant. Note that $S_{\mathbb{R}}^{H}(z) \simeq H / M$ as manifolds.

1.6. Invariant measure on $Y$. Lemma 1.8 allows for a natural normalization of the invariant measure on $Y$. Assume that invariant measures on $G, A$ and $N$ have been fixed and let $\mathcal{W}_{H}=N_{K \cap H}(\mathfrak{a}) / Z_{H \cap K}(\mathfrak{a})$ be the small Weyl group. By Lemma 1.8 the union

$$
\mathcal{U}=\bigcup_{w \in \mathcal{W} / \mathcal{W}_{H}} A N w \cdot y_{o}
$$

is disjoint open and dense in $Y$. As the complement is an analytic set, it follows that $Y \backslash \mathcal{U}$ has measure zero. According to [18, Lemma 7.3, we now can normalize the invariant measure on $Y$ such that for all $f \in L^{1}(Y)$ :

$$
\int_{Y} f(y) d y=\sum_{w \in \mathcal{W} / \mathcal{W}_{H}} \int_{A} \int_{N} f\left(\text { anw } \cdot y_{o}\right) d a d n .
$$

\section{The FreChÉt MOdule $C R^{\infty}\left(\Xi_{+}\right)$}

In this section we use the right $A$-action on $\Xi_{\mathbb{R}}$ and $\Xi_{+}$to define $G$-submodules of the smooth $A$-covariant functions on $\Xi_{\mathbb{R}}$ respectively $C R$-functions on $\Xi_{+}$. Those modules are the standard realization respectively a CR-realization of the space of smooth vectors in the principal series representations given by induction from the right. Note that later we will use the induction from the left. 
Recall, that $A$ acts on the space of horospheres from the right. This action induces a right regular representation of $A$ on any function space on $\Xi_{\mathbb{R}}, \Xi_{+}$or any other right invariant set of horospheres given by

$$
(R(a) f)(\xi)=f(\xi \cdot a)
$$

Let $\rho=1 / 2 \sum_{\alpha \in \Sigma^{+}}\left(\operatorname{dim} \mathfrak{g}^{\alpha}\right) \cdot \alpha$. The index ${ }_{\lambda}$ will denote the subspace of $(\lambda-\rho)$-covariant functions. In particular

$$
C^{\infty}\left(\Xi_{\mathbb{R}}\right)_{\lambda}=\left\{f \in C^{\infty}\left(\Xi_{\mathbb{R}}\right) \mid R(a) f=a^{\lambda-\rho} f\right\} .
$$

We recall that $G$ acts $C^{\infty}\left(\Xi_{\mathbb{R}}\right)$ by left translations in the argument

$$
(L(g) f)(\xi)=f\left(g^{-1} \cdot \xi\right)
$$

for $g \in G, f \in C^{\infty}\left(\Xi_{\mathbb{R}}\right)$ and $\xi \in \Xi_{\mathbb{R}}$. The so obtained representation $\left(L, C^{\infty}\left(\Xi_{\mathbb{R}}\right)_{\lambda}\right)$ is the smooth model of the spherical principal series with parameter $\lambda$. Similarly

$$
C R^{\infty}\left(\Xi_{+}\right)_{\lambda}=\left\{f \in C^{\infty}\left(\Xi_{+}\right) \mid R(a) f=a^{\lambda-\rho} f\right\} .
$$

As characters on $A$ extend to holomorphic functions on $T\left(\Omega_{H}\right)$, it follows that the restriction map

$$
\operatorname{Res}_{\lambda}: C R^{\infty}\left(\Xi_{+}\right)_{\lambda} \rightarrow C^{\infty}\left(\Xi_{\mathbb{R}}\right)_{\lambda},\left.\quad f \mapsto f\right|_{\Xi_{\mathbb{R}}}
$$

is a $G$-equivariant topological isomorphism of $G$-modules.

2.1. $C R$-realization of the $H$-spherical holomorphic vector. For each $\lambda \in \mathfrak{a}_{\mathbb{C}}^{*}$ we define a certain $H$-invariant element $f_{\lambda} \in C R^{-\infty}\left(\Xi_{+}\right)_{\lambda}$ which was called the $H$-spherical holomorphic distribution vector in [9]. The generalized function $f_{\lambda}$ is defined by

$$
f_{\lambda}(\xi)=a\left(\xi^{-1} z_{H}^{-1}\right)^{\rho-\lambda} \quad\left(\xi \in \Xi_{+}\right) .
$$

We notice that on the dense subset

$$
\Xi_{+}^{\prime}=\bigcup_{w \in \mathcal{W}} H w T\left(\Omega_{H}\right) \cdot \xi_{o}
$$

of $\Xi_{+}$, the function belongs to $C R^{\infty}\left(\Xi_{+}^{\prime}\right)_{\lambda}$ and is given by

$$
f_{\lambda}\left(h w a \cdot \xi_{0}\right)=\left(w^{-1} z_{H} w\right)^{\lambda-\rho} a^{\lambda-\rho} .
$$

For $\Re \lambda \ll 0$, this function is actually continuous on $\Xi_{+}$and the meromorphic continuation in $\lambda$ as a distribution is achieved with Bernstein's theorem [3. There are no singularities on the imaginary axis $i \mathfrak{a}^{*}$ and we arrive at a well defined analytic assignment

$$
\lambda \mapsto C R^{-\infty}\left(\Xi_{+}\right)_{\lambda}^{H}, \lambda \mapsto f_{\lambda}
$$

(cf. 9], Th. 2.4.1). 


\section{THE HOLOMORPHIC HOROSPHERICAL RADON TRANSFORM}

The real Radon transform on $X$ is a $G$-equivariant injective map

$$
\mathcal{R}_{\mathbb{R}}: \mathcal{S}(X) \rightarrow C^{\infty}\left(\Xi_{\mathbb{R}}\right)
$$

The purpose of this section is to show that $\mathcal{R}_{\mathbb{R}}$ has a natural extension to a $G$-equivariant map

$$
\mathcal{R}: \mathcal{H}^{2}(D)_{0} \rightarrow C R^{\infty}\left(\Xi_{+}\right)
$$

which we call the holomorphic horospherical Radon transform. Here $\mathcal{H}^{2}(D)_{0} \hookrightarrow L^{2}(X)$ is a dense a subspace of the most-continuous Hardy space $\mathcal{H}^{2}(D) \subset \mathcal{O}(D)$ of $L^{2}(Y)$ (cf. [9]). On the infinnitesimal level this extension is related to the previously established fact (2.1), i.e. $C^{\infty}\left(\Xi_{\mathbb{R}}\right)_{\lambda}$ is canonically $G$-isomorphic to $C R^{\infty}\left(\Xi_{+}\right)_{\lambda}$ via restriction.

This section is organized as follows: First we have to recall some facts about the Fourier analysis on $X$, in particular Arthur's spectral characterization of the Schwartz space $\mathcal{C}(X)$. Subsequently we give a brief summary on the most-continuous Hardy space $\mathcal{H}^{2}(D)$ of $[9$. Finally we define the holomorphic horospherical Radon transform $\mathcal{R}$ and discuss some of its properties.

3.1. Fourier analysis on $X$. We recall the compact realization of the principal series representations. Let $B=M \backslash K$. For $\lambda \in \mathfrak{a}_{\mathbb{C}}^{*}$ define a representation $\pi_{\lambda}$ of $G$ on $L^{2}(B)$ by

$$
\pi_{\lambda}(g) f(M k)=a(k g)^{\rho-\lambda} f(M k(k g)) .
$$

Then $\left(\pi_{\lambda}, L^{2}(B)\right)$ is unitary if $\lambda \in i \mathfrak{a}^{*}$. We write $\mathcal{H}_{\lambda}=L^{2}(B)$ to indicate the dependence of the $G$-action on $\lambda$. Let $\mathfrak{a}^{+}=\left\{H \in \mathfrak{a} \mid\left(\forall \alpha \in \Delta^{+}\right) \alpha(H)>0\right\}$ and

$$
\mathfrak{a}_{+}^{*}=\left\{\lambda \in \mathfrak{a} \mid\left(\forall H \in \mathfrak{a}^{+}\right) \lambda(H)>0\right\} .
$$

Denote by $\hat{G}_{\mathrm{r}}$ the reduced dual of $G$ and by $\hat{G}_{\text {rsp }}$ the spherical reduced dual. Then $i \mathfrak{a}_{+}^{*} \ni$ $\lambda \mapsto\left[\pi_{\lambda}\right] \in \hat{G}_{\mathrm{rsp}}$ is an isomorphism of measure spaces. Here $\left[\pi_{\lambda}\right]$ denotes the equivalence class of $\pi_{\lambda}$. We have

$$
L^{2}(X) \simeq \int_{i \mathfrak{a}_{+}^{*}}^{\oplus} \mathcal{H}_{\lambda} \frac{d \lambda}{|\mathbf{c}(\lambda)|^{2}}
$$

where $\mathbf{c}(\lambda)$ is the Harish-Chandra $c$-function. To explain the above isomorphism, we need some basic fact on the Fourier transform on $X$. For that, recall that $a: X \rightarrow A$ denotes the $A$-projection with regard to the Iwasawa isomorphism $X=N A \cdot x_{o} \simeq N \times A$. Set $\mathcal{X}=B \times i \mathfrak{a}_{+}^{*}$ and define a Radon measure $d \mu_{\mathcal{X}}$ on $\mathcal{X}$ by

$$
d \mu_{\mathcal{X}}(b, \lambda):=d b \frac{d \lambda}{|\mathbf{c}(\lambda)|^{2}}
$$


For $f \in L^{1}(X) \cap L^{2}(X)$ define its spherical Fourier transform $\hat{f}: \mathcal{X} \rightarrow \mathbb{C}$ by

$$
\hat{f}(b, \lambda)=\int_{X} f(x) a(b x)^{\rho-\lambda} d x .
$$

We will also write $\mathcal{F}_{X}(f)$ for $\hat{f}$. We can normalize the left-Haar measure $d x$ on $G$ such that the Fourier transform extends to an unitary isomorphism ${ }^{\wedge}: L^{2}(G) \rightarrow L^{2}(\mathcal{X}, d \mu \mathcal{X})$. If $f$ is rapidly decreasing (see exact definition in a moment), then the Fourier inversion formula holds pointwise:

$$
f(x)=\int_{\mathcal{X}} \hat{f}(b, \lambda) a(b x)^{\rho+\lambda} d \mu_{\mathcal{X}}(b, \lambda) \quad(x \in X) .
$$

For $\lambda \in i \mathfrak{a}^{*}$ define $\hat{f}_{\lambda} \in L^{2}(B)$ by $b \mapsto \hat{f}_{\lambda}(b)=\hat{f}(b, \lambda)$. Then the isomorphism in (3.2) is given by

$$
L^{2}(X) \ni f \mapsto\left(\hat{f}_{\lambda}\right)_{\lambda} \in \int_{i \mathfrak{a}_{+}^{*}}^{\oplus} \mathcal{H}_{\lambda} \frac{d \lambda}{|\mathbf{c}(\lambda)|^{2}} .
$$

In the following we will also need the operator valued Fourier transform. If $\mathcal{H}$ is a Hilbert space, then $B_{2}(\mathcal{H}) \simeq \mathcal{H} \hat{\otimes} \mathcal{H}^{*}$ denotes the Hilbert space of Hilbert-Schmidt operators on $\mathcal{H}$. Write

$$
L^{2}(G)_{\mathrm{sph}}=\int_{i \mathfrak{a}_{+}^{*}}^{\oplus} B_{2}\left(\mathcal{H}_{\lambda}\right) \frac{d \lambda}{|\mathbf{c}(\lambda)|^{2}}
$$

for the $K$-spherical spectrum in $L^{2}(G)$. Recall that the isomorphism is given by the operator valued Fourier transform $\mathcal{F}(f)(\lambda)=\int_{G} f(x) \pi_{\lambda}(x) d x, f \in L^{1}(G) \cap L^{2}(G)$. The inverse map is

$$
f(g)=\int_{i \mathfrak{a}_{+}^{*}} \operatorname{Tr}\left(\pi_{\lambda}\left(g^{-1}\right) \mathcal{F}(f)(\lambda)\right) \frac{d \lambda}{|\mathbf{c}(\lambda)|^{2}} .
$$

The constant function $v_{K, \lambda}=\mathbf{1}_{B}$ defines a normalized $K$-fixed vector in $\mathcal{H}_{\lambda}$. Assume that $f \in L^{1}(G) \cap L^{2}(G)_{\mathrm{sph}}$. Then, because $\mathcal{F}(f)(\lambda)=\mathcal{F}\left(R_{k} f\right)(\lambda)=\mathcal{F}(f)(\lambda) \pi_{\lambda}(k)$, it follows, that

$$
\mathcal{F}(f)(\lambda) v=\left\langle v, v_{K, \lambda}\right\rangle \mathcal{F}(f)(\lambda) v_{K, \lambda}=\left\langle v, v_{K, \lambda}\right\rangle \hat{f}_{\lambda} .
$$

For $x=k_{1} \exp (Z) k_{2} \in G$, with $Z \in \mathfrak{a}$ and $k_{1}, k_{2} \in K$, let $\sigma(x)=-B(Z, d \theta(\mathbf{1})(\mathbf{Z}))$, where $B$ is the Killing form on $\mathfrak{g}$. Denote by $U(\mathfrak{g})$ the Universal enveloping algebra of $\mathfrak{g}$ and by $\varphi_{0}$ the basic spherical function. For $D, E \in U(\mathfrak{g}), s \in \mathbb{R}$ and $f \in C^{\infty}(G)$, let

$$
p_{D, E, s}(f):=\sup _{x \in G}\left|L_{D} R_{E} f(x)\right| \varphi_{0}(x)^{-1}(1+\sigma(x))^{s} .
$$

Then $\mathcal{C}(G)$ is the space of smooth functions on $G$ such that $p_{D, E, s}(f)<\infty$ for all such $E, D$ and $s$, cf. [12].

We set

$$
\mathcal{C}(G)_{\mathrm{sph}}=\mathcal{C}(G) \cap L^{2}(G)_{\mathrm{sph}}
$$

Let $\mathcal{S}\left(i \mathfrak{a}_{+}^{*}\right)=\left\{\left.f\right|_{i \mathfrak{a}_{+}^{*}} \mid f \in \mathcal{S}\left(i \mathfrak{a}^{*}\right)\right\}$. We recall a theorem of Arthur [1], p. 4719: 
Theorem 3.1. The operator valued Fourier transfrom $\mathcal{F}$ maps $\mathcal{C}(G)_{\mathrm{sph}}$ isomorphically onto

$$
\left\{A(\cdot) \in \int_{i \mathfrak{a}_{+}^{*}}^{\oplus} B_{2}\left(\mathcal{H}_{\lambda}\right) \frac{d \lambda}{|\mathbf{c}(\lambda)|^{2}} \mid\left(\forall v, w \in L^{2}(B)_{\mathrm{K}-\mathrm{fin}}\right)\langle A(\cdot) v, w\rangle \in \mathcal{S}\left(i \mathfrak{a}_{+}^{*}\right)\right\} .
$$

3.2. The most-continuous Hardy space. We recall now the spectral definition of the Hardy space $\mathcal{H}^{2}(D)$ from [9]. For $v \in \mathcal{H}_{\lambda}$ define an analytic function $f_{v, \lambda}$ on $X$ by

$$
f_{v, \lambda}(x)=\left\langle\pi_{\lambda}\left(x^{-1}\right) v, v_{K, \lambda}\right\rangle=\left\langle v, \pi_{\lambda}(x) v_{K, \lambda}\right\rangle
$$

and recall that $f_{v, \lambda}$ extends to a holomorphic function $\tilde{f}_{v, \lambda}$ on $D$ via

$$
\tilde{f}_{v, \lambda}(x)=\left\langle v, \pi_{\lambda}(\bar{x}) v_{K, \lambda}\right\rangle
$$

for $x \in D$, cf. 9], Proposition 2.2.3. In particular

$$
f_{v, \lambda}\left(g a \cdot x_{o}\right)=\left\langle\pi_{\lambda}\left(g^{-1}\right) v, \pi_{\lambda}\left(a^{-1}\right) v_{K, \lambda}\right\rangle
$$

for $g \in G$ and $a \in \exp \left(i \Omega_{H}\right)$

Define a generalized hyperbolic cosine function on $i \mathfrak{a}^{*}$ by

$$
\cosh (\lambda)=\sum_{w \in \mathcal{W} / \mathcal{W}_{H}} z_{H}^{2 w^{-1} \lambda}
$$

for $\lambda \in i \mathfrak{a}^{*}$. Define a measure $\mu$ on $i \mathfrak{a}_{+}^{*}$ by

$$
d \mu(\lambda)=\frac{d \lambda}{\cosh (\lambda) \cdot|\mathbf{c}(\lambda)|^{2}}
$$

With this preparation we can define the unitary representation $\left(L, \mathcal{H}^{2}(D)\right)$ of $G$ by

$$
\left(L, \mathcal{H}^{2}(D)\right)=\int_{i \mathfrak{a}_{+}^{*}}^{\oplus}\left(\pi_{\lambda}, \mathcal{H}_{\lambda}\right) d \mu(\lambda) .
$$

Thus $\mathcal{H}^{2}(D)$ is the Hilbert space of all measurable functions $s: i \mathfrak{a}_{+}^{*} \rightarrow L^{2}(M \backslash K)$ such that $\|s\|^{2}=\int_{i \mathfrak{a}_{+}^{*}}\|s(\lambda)\|^{2} d \mu(\lambda)<\infty$. In the sequel we write often write $s_{\lambda}$ for $s(\lambda)$. We will also write $\|\cdot\|_{H}$ for the norm on $\mathcal{H}^{2}(D)$. Recall from [9] that the map

$$
\Phi: \mathcal{H}^{2}(D) \hookrightarrow \mathcal{O}(D), \quad s=\left(s_{\lambda}\right) \mapsto\left(x \mapsto \int_{i \mathfrak{a}_{+}^{*}} \tilde{f}_{s_{\lambda}, \lambda}(x) d \mu(\lambda)\right)
$$

is a $G$-equivariant continuous injection. In the sequel we view $\mathcal{H}^{2}(D)$ as a subspace of $\mathcal{O}(D)$ and call $\mathcal{H}^{2}(D)$ the most-continuous Hardy space of $Y$. This notion is motivated by the main result of [9] which states that there exists an isometric $G$-equivariant boundary value mapping

$$
b: \mathcal{H}^{2}(D) \rightarrow L_{\mathrm{mc}}^{2}(Y), \quad f \mapsto b(f) .
$$


3.3. The Fourier Transform on $X$ and the Hardy space. The definition of $\mathcal{H}^{2}(D)$ in the previous subsection does not use the Fourier transform on $X$. But the following Lemma shows that the space $\mathcal{H}^{2}(D)$ has a natural description in terms of the Fourier transform.

Lemma 3.2. Let $f \in \mathcal{H}^{2}(D)$. Then the following hold:

(1) $\left.f\right|_{X} \in L^{2}(X)$ and

$$
\begin{aligned}
f(x) & =\int_{i \mathrm{a}_{+}^{*}} \hat{f}(b, \lambda) \cosh (\lambda) a(b x)^{\lambda+\rho} d \mu(b, \lambda) \\
\|f\|_{H}^{2} & =\int_{\mathcal{X}}|\hat{f}(b, \lambda)|^{2} \cdot \cosh (\lambda) d \mu_{\mathcal{X}}(b, \lambda) \geq\|f\|_{L^{2}(X)}^{2} .
\end{aligned}
$$

(2) Let $f=\Phi^{-1}\left(s_{\lambda}\right) \in \mathcal{H}^{2}(D)$. Then

$$
\hat{f}_{\lambda}=\frac{s_{\lambda}}{\cosh (\lambda)} .
$$

(3) For $a=\exp (i Y) \in \exp \left(i \Omega_{H}\right)$ let $f_{a}: G \rightarrow \mathbb{C}$ by $f_{a}(g)=\tilde{f}\left(g a \cdot x_{o}\right)$. Let $Q \subset \exp \left(i \Omega_{H}\right)$ be compact and such that $a \in Q$. Then there exists a constant $C_{Q}>0$ such that

$$
\left\|f_{a}\right\|_{L^{2}(G)} \leq C_{Q}\|f\|_{H}
$$

Proof. (1) and (2) Let $f \in \mathcal{H}^{2}(D)$ and $f=\int_{i_{\mathfrak{a}_{+}^{*}}} s_{\lambda} d \mu(\lambda)$. Then obviously we have (2), i.e.,

$$
f=\int_{i \mathbf{a}_{+}^{*}} \frac{s_{\lambda}}{\cosh (\lambda)} \frac{d \lambda}{|\mathbf{c}(\lambda)|^{2}}
$$

and

$$
\begin{aligned}
\|f\|_{H}^{2} & =\int_{i \mathfrak{a}_{+}^{*}}\left\|s_{\lambda}\right\|_{L^{2}(B)}^{2} d \mu(\lambda) \\
& =\int_{i \mathfrak{a}_{+}^{*}}\left\|\frac{s_{\lambda}}{\cosh (\lambda)}\right\|_{L^{2}(B)}^{2} \cosh (\lambda) \frac{d \lambda}{|\mathbf{c}(\lambda)|^{2}} \\
& \geq \int_{i \mathfrak{a}_{+}^{*}}\left\|\frac{s_{\lambda}}{\cosh (\lambda)}\right\|_{L^{2}(B)}^{2} \frac{d \lambda}{|\mathbf{c}(\lambda)|^{2}} \\
& =\|f\|_{L^{2}(X)}^{2} .
\end{aligned}
$$

Thus $f \in L^{2}(X)$ and we can write $f=\int_{\mathcal{X}} \hat{f}(b, \lambda) a(b \cdot)^{\rho+\lambda} d b \frac{d \lambda}{|\mathbf{c}(\lambda)|^{2}}$. Equation (3.6) implies that $\hat{f}(b, \lambda)=s_{\lambda}(b) / \cosh (\lambda)$ or $s_{\lambda}(b)=\hat{f}(b, \lambda) \cosh (\lambda)$ for almost all $\lambda$. This finish the proof of (1).

(2) We recall Faraut's version of the Gutzmer identity [5]

$$
\int_{G}\left|f\left(g a \cdot x_{o}\right)\right|^{2} d g=\int_{\mathcal{X}}|\hat{f}(b, \lambda)|^{2} \varphi_{\lambda}\left(a^{2}\right) d b \frac{d \lambda}{|\mathbf{c}(\lambda)|^{2}}
$$


where $\varphi_{\lambda}\left(a^{2}\right)$ is the analytically continued spherical function given by

$$
\varphi_{\lambda}\left(a^{2}\right)=\int_{K}\left|a(k a)^{\rho+\lambda}\right|^{2} d k
$$

Now for a compact subset $Q \subset \exp \left(i \Omega_{H}\right)$ there exists a constant $C_{Q}>0$ such that

$$
\varphi_{\lambda}\left(a^{2}\right) \leq C_{Q} \cosh (\lambda)
$$

for all $\lambda$ (cf. [15], Lemma 2.1) and the assertion of the lemma follows.

In order to define the Radon transform for functions in the Hardy space we first need a technical fact, interesting on its own.

Let $\left(\int_{i \mathfrak{a}_{+}^{*}}^{\oplus} \mathcal{H}_{\lambda} d \mu(\lambda)\right)_{0}$ denote the space of all sections such that for all $v \in L^{2}(B)$

$$
i \mathfrak{a}_{+}^{*} \ni \lambda \mapsto\left\langle s_{\lambda}, v\right\rangle \in S\left(i \mathfrak{a}_{+}^{*}\right) .
$$

Then we set

$$
\mathcal{H}^{2}(D)_{0}=\Phi^{-1}\left(\left(\int_{i \mathfrak{a}_{+}^{*}}^{\oplus} \mathcal{H}_{\lambda} d \mu(\lambda)\right)_{0}\right) .
$$

Theorem 3.3. Let $f \in \mathcal{H}^{2}(D)_{0}$. Fix $z \in T\left(\Omega_{H}\right)$. Then the function

$$
G \ni g \mapsto f\left(g z \cdot x_{o}\right) \in \mathbb{C}
$$

belongs to $\mathcal{C}(G)$. Moreover, the following functions are locally bounded on $T\left(\Omega_{H}\right)$ :

(1) $z \rightarrow \int_{G}\left|f\left(g z \cdot x_{o}\right)\right|^{2} d g$

(2) $z \rightarrow \int_{N}\left|f\left(n z \cdot x_{o}\right)\right| d n$

Proof. Without loss of generality we may assume that $z=a \in \exp \left(i \Omega_{H}\right)$. We identify $f$ with a right $K$-invariant function on $G$. Let $v, w \in \mathcal{H}_{\lambda}$, then we have by (3.3) and Lemma 3.2

$$
\begin{aligned}
\left\langle\mathcal{F}\left(R_{a} f\right)(\lambda) v, w\right\rangle & =\left\langle\mathcal{F}(f)(\lambda) \pi_{\lambda}(a) v, w\right\rangle \\
& =\left\langle\pi_{\lambda}(a) v, v_{K, \lambda}\right\rangle\left\langle\hat{f}_{\lambda}, v\right\rangle \\
& =\frac{\left\langle\pi_{\lambda}(a) v, v_{K, \lambda}\right\rangle}{\cosh (\lambda)}\left\langle s_{\lambda}, v\right\rangle .
\end{aligned}
$$

Let $Q$ be a compact subset of $\exp \left(i \Omega_{H}\right), a \in Q$. Let $\mathcal{D}$ be a constant coefficient differential operator on $i \mathfrak{a}^{*}$. Then there exists a polynomial $p$ such that

$$
\mathcal{D}\left\langle\pi_{\lambda}(a) v, v_{K, \lambda}\right\rangle=\int_{K} p\left(<\rho+\lambda, \log \left(k a^{-1}\right)>\right) v(k) v_{K,-\lambda}\left(k a^{-1}\right) d k .
$$

Hence, for all such $\mathcal{D}$ we get:

$$
\sup _{a \in Q}\left|\mathcal{D} \frac{\left\langle\pi_{\lambda}(a) v, v_{K, \lambda}\right\rangle}{\cosh (\lambda)}\right| \leq C_{Q}
$$


for some constant $C_{Q}>0$. As $\lambda \mapsto\left\langle s_{\lambda}, v\right\rangle$ is rapidly decreasing it follows that $\lambda \mapsto$ $\left\langle\mathcal{F}\left(R_{a} f\right)(\lambda) v, w\right\rangle$ is rapidly decreasing and hence $R_{a} f \in \mathcal{C}(G)$ by Theorem 3.1 .

Statement (1) is Lemma 3.2, part 3 and part (2) follows from Lemma 22 in 12

3.4. The definition of the Radon Transform. Denote by $C R\left(\Xi_{+}\right)$the vector space of continuous $C R$-functions on $\Xi_{+}$, i.e. the space of continuous functions on $\Xi_{+} \simeq K / M \times$ $T\left(\Omega_{H}\right)$ (cf. (1.13) ) which are holomorphic in the second variable.

Lemma 3.4. Let $f \in \mathcal{H}^{2}(D)_{0}$. Then the assignment

$$
\Xi_{+} \ni \xi=g a \cdot \xi_{o} \mapsto a^{-2 \rho} \int_{N} f\left(g n a \cdot x_{o}\right) d n \in \mathbb{C}
$$

defines a $C R$-function on $\Xi_{+}$.

Proof. It follows from Theorem 3.3 that the right hand side is a continuous function. It remains to show that is a $C R$-function. For that let $g=k b$ for $k \in K$ and $b \in A$. The right hand side becomes

$$
a^{-2 \rho} \int_{N} f\left(k b n a \cdot x_{o}\right) d n=(a b)^{-2 \rho} \int_{N} f\left(k n b a \cdot x_{o}\right) d n
$$

and the holomorphicity in $a b$ follows with Theorem 3.3 .

In view of this lemma, the prescription

$$
\mathcal{R}: \mathcal{H}^{2}(D)_{0} \rightarrow C R\left(\Xi_{+}\right), \quad f \mapsto\left(\xi=g a \cdot \xi_{o} \mapsto a^{-2 \rho} \int_{N} f\left(g n a \cdot x_{o}\right) d n\right)
$$

is a well defined and continuous $G$-equivariant map. We call $\mathcal{R}$ the holomorphic horospherical Radon transform.

Remark 3.5. (a) Notice that $E(\xi) \cap D$ for $\xi \in \Xi_{+}$contains the real horosphere $E_{\mathbb{R}}(\xi)$. The holomorphic Radon transform $\mathcal{R}$ then writes as

$$
\mathcal{R}(f)(\xi)=\int_{E_{\mathbb{R}}(\xi)} f\left(\xi^{\prime}\right) d \nu_{\xi}\left(\xi^{\prime}\right)
$$

with $d \nu_{\xi}$ the $a^{-2 \rho_{\text {-times }}}$ the measure on $E_{\mathbb{R}}(\xi)$ obtained by the natural identification of the real horosphere $E_{\mathbb{R}}(\xi)$ with $N$. It is clear that any other $N$-orbit in $E(\xi) \cap D$ would yield the same result.

(b) If $a \in A$, then

$$
a^{-2 \rho} \int_{N} f\left(\text { gna } \cdot x_{o}\right) d n=\int_{N} f\left(\text { gan } \cdot x_{o}\right) d n
$$

and hence the holomorphic Radon transform agrees with the usual Radon transform on $X$.

If the function $f \in \mathcal{H}^{2}(D)_{0}$ is left $K$-invariant, then we can define the holomorphic Abeltransform by: 


$$
\mathcal{A}(f)(z)=z^{-\rho} \int_{N} f\left(n z \cdot x_{o}\right) d n \quad\left(z \in T\left(\Omega_{H}\right)\right) .
$$

Note that $\mathcal{A}$ is just the restriction of the holomorphic Radon transform to $K$-invariant function (modulo the $z^{-\rho}$-factor). Further let us remark that $\mathcal{A}$ gives a continuous mapping

$$
\mathcal{A}: \mathcal{H}^{2}(D)_{0}^{K} \rightarrow \mathcal{O}\left(T\left(\Omega_{H}\right)\right)^{\mathcal{W}}, \quad \mathcal{A}(f)(z)=z^{-\rho} \int_{N} f\left(n z \cdot x_{o}\right) d n
$$

\section{The holomorphic Radon transform as Cauchy integral I: The HYPERBOLOID}

In this and the next section we will show (for an appropriate class of $Y$ 's) that the holomorphic Radon transform on the NCC space $Y$ can be expressed as a Cauchy type integral. For that purpose it is instructive to explaining the example of the hyperboloid first. For earlier treatments of the hyperboloid with alternative methods we refer to [6], [7]. We start by recalling some standard function spaces on $Y$.

4.1. Function spaces. Let $Y$ be a NCC space. For $g \in G$ let $\Theta(g)=\varphi_{0}\left(g \tau(g)^{-1}\right)^{1 / 2}$. Then $\Theta$ is left $K$-invariant and right $H$-invariant. For $g=k \exp (Z) h$ with $Z \in \mathfrak{a}$ define $\left\|g \cdot y_{0}\right\|:=\|Z\|$, where $\|Z\|=\sqrt{\operatorname{Tr} \operatorname{ad}(Z)^{2}}$. Let $D \in U(\mathfrak{g})$, were $U(\mathfrak{g})$ is the enveloping algebra of $\mathfrak{g}_{\mathbb{C}}$, and view $L_{D}$ as a differential operator on $Y$. For $n \in \mathbb{N}$ and $f \in C^{\infty}(Y)$ define

$$
p_{n, D}(f)=\sup _{y \in Y} \Theta_{G}(y)^{-1}(1+\|y\|)^{n}\left|L_{D} f(y)\right| .
$$

Then the Schwartz space $\mathcal{C}(Y)$ is defined as the space of smooth function on $Y$ such that $p_{n, D}(f)<\infty$ for all $n$ and $D$. It is well known, that $\mathcal{C}(Y) \subset L^{2}(Y)$, but $\mathcal{C}(Y)$ is not contained in $L^{1}(Y)$. We will therefore need a smaller space to make sure that the Cauchy integral exists. For that, define for $r>0$ the space

$$
\mathcal{S}_{r}(Y):=\left\{f \in C^{\infty}(Y)\left|(\forall D \in U(\mathfrak{g})) \sup _{y \in Y} e^{r\|y\|}\right| L_{D} f(y) \mid<\infty\right\}
$$

and

$$
\mathcal{S}(Y):=\bigcap_{r>0} \mathcal{S}_{r}(Y)
$$

Then $\mathcal{S}(Y) \subset L^{1}(Y) \cap L^{2}(Y)$ and $C_{c}^{\infty}(Y) \subset \mathcal{S}(Y) \subset \mathcal{C}(Y)$. The space $\mathcal{S}(Y)$ is called the (zero) Schwartz space, cf. [4. It follows from Theorem 3 in 4 and our spectral definition of the space $\mathcal{H}^{2}(D)$ that $\mathcal{H}^{2}(D)_{00}:=\mathcal{H}^{2}(D)_{K \text {-finite }} \cap \mathcal{S}(Y) \neq\{0\}$ and is dense in $\mathcal{H}^{2}(D)$. Here we have used that the elements in $\mathcal{H}^{2}(D)_{K \text {-finite }}$ have boundary values on $Y$ (cf. [9], Sect. 3). In particular we have that every element $f \in \mathcal{H}^{2}(D)_{00}$ is integrable on $Y$, has a holomorphic extension to $D$, and that the integral $\int_{N} f\left(a n w \cdot y_{0}\right) d n$ is well defined for all $a \in A$ and $w \in \mathcal{W}$. We will use this without comments in the following. 
4.2. The Radon transform and Cauchy integral on the hyperboloid. Assume that $n \geq 2$ and let $G=\mathrm{SO}_{e}(1, n)$ be the Lorentz group. Let us fix our choices for the groups $A$, $N$ and $K$. For the maximal compact subgroup we take

$$
K=\left\{k_{R}=\left(\begin{array}{ll}
1 & 0 \\
0 & R
\end{array}\right) \mid R \in \mathrm{SO}(n)\right\} \simeq \mathrm{SO}(n) .
$$

Next, for $z \in \mathbb{C}$ we set

$$
a_{z}=\left(\begin{array}{ccc}
\cosh z & 0 & \sinh z \\
0 & \mathbf{1}_{n-1} & 0 \\
\sinh z & 0 & \cosh z
\end{array}\right)
$$

and

$$
A=\left\{a_{t} \mid t \in \mathbb{R}\right\} \quad \text { and } \quad A_{\mathbb{C}}=\left\{a_{z} \mid z \in \mathbb{C}\right\} .
$$

Note that $\mathfrak{a}=\mathbb{R} Z$ and $a_{z}=\exp (z Z)$ with $Z=E_{1 n+1}+E_{n+11}$. The only positive root is $\alpha$, determined by $\alpha(Z)=1$ and hence $Z_{H}=\frac{\pi}{2} Z$. We also have, that $\rho=\frac{n-1}{2} \alpha$.

Further, for $v \in \mathbb{C}^{n-1}$ and $(v, v)=\sum_{j=1} v_{j} v_{j}$ we define an unipotent matrix

$$
n_{v}=\left(\begin{array}{ccc}
1+\frac{1}{2}(v, v) & v^{T} & -\frac{1}{2}(v, v) \\
v & \mathbf{1}_{n-1} & -v \\
\frac{1}{2}(v, v) & v^{T} & 1-\frac{1}{2}(v, v)
\end{array}\right) .
$$

Then $N$ and $N_{\mathbb{C}}$ are given by

$$
N=\left\{n_{v} \mid v \in \mathbb{R}^{n-1}\right\} \quad \text { and } \quad N_{\mathbb{C}}=\left\{n_{v} \mid v \in \mathbb{C}^{n-1}\right\} .
$$

Define a quadratic form

$$
z, w \mapsto z \cdot w=z_{0} w_{0}-\sum_{j=1}^{n} z_{j} w_{j}
$$

on $\mathbb{C}^{n+1}$ and let

$$
\square(\mathbf{z})=z_{0}^{2}-z_{1}^{2}-\ldots-z_{n}^{2} \quad\left(\mathbf{z}=\left(z_{0}, \ldots, z_{n}\right)^{T} \in \mathbb{C}^{n+1}\right)
$$

be the corresponding quadratic form. We define the real and complex hyperboloids by

$$
X=\left\{\mathbf{x} \in \mathbb{R}^{n+1} \mid \square(\mathbf{x})=1, x_{0}>0\right\}
$$

and

$$
X_{\mathbb{C}}=\left\{\mathbf{z} \in \mathbb{C}^{n+1} \mid \square(\mathbf{z})=1\right\} .
$$

As a common base point for $X$ and $X_{\mathbb{C}}$ we take $\mathbf{x}_{o}=(1,0, \ldots, 0)^{T}$ and note that the map

$$
G_{\mathbb{C}} / K_{\mathbb{C}} \rightarrow X_{\mathbb{C}}, \quad g K_{\mathbb{C}} \mapsto g\left(\mathbf{x}_{o}\right)
$$

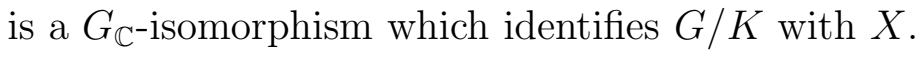


We have that $a_{z} \cdot \mathbf{x}_{o}=(\cosh (z), 0, \ldots, 0, \sinh (z))^{T}$ and hence $\mathbf{y}_{0}=z_{H} \cdot \mathbf{x}_{o}=(0, \ldots, 0, i)^{T}$. It is clear that the stabilizer of $\mathbf{y}_{o}$ is

$$
H=\left\{\left(\begin{array}{ll}
h & 0 \\
0 & 1
\end{array}\right) \mid h \in \mathrm{SO}_{e}(1, n-1)\right\} \simeq \mathrm{SO}_{e}(1, n-1) .
$$

We have therefore with this identification:

$$
D=\left\{\mathbf{z}=\mathbf{x}+i \mathbf{y} \in X_{\mathbb{C}} \mid \square(\mathbf{x})>0, x_{0}>0\right\}
$$

and

$$
Y=G\left(\mathbf{y}_{0}\right)=\left\{i \mathbf{y} \in i \mathbb{R}^{n+1} \mid \square(\mathbf{y})=-1\right\}
$$

Set

$$
\Xi=\left\{\zeta \in \mathbb{C}^{n+1} \mid \zeta \neq 0, \square(\zeta)=0\right\} .
$$

If $\xi_{o}=(1,0, \ldots, 0,1)^{T} \in \Xi$, then the stabilizer of $\xi_{o}$ is $M_{\mathbb{C}} N_{\mathbb{C}}$ and the map

$$
G_{\mathbb{C}} / M_{\mathbb{C}} N_{\mathbb{C}} \rightarrow \Xi, \quad g M_{\mathbb{C}} N_{\mathbb{C}} \mapsto g \cdot \xi_{o}
$$

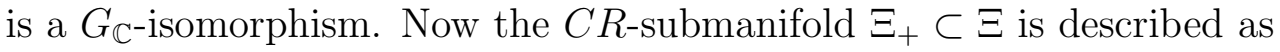

$$
\begin{aligned}
\Xi_{+} & =G\left\{\left(e^{i t}, 0, \ldots, 0, e^{i t}\right)^{T}|| t \mid<\frac{\pi}{2}, t \in \mathbb{R}\right\} \\
& =\{\zeta=\xi+i \eta \in \Xi: \square(\xi)=\square(\eta)=0 ; \xi \neq 0\}_{0} .
\end{aligned}
$$

We will also use certain $G$-subdomains of $\Xi_{+}$: for $0<c \leq \frac{\pi}{2}$ set

$$
\Xi_{c}=G\left\{\left(e^{i t}, 0, \ldots, 0, e^{i t}\right)^{T}|| t \mid<c, t \in \mathbb{R}\right\} .
$$

In order to define the Cauchy-transform we need to establish a simple, but important, technical fact.

Lemma 4.1. For all $\xi \in \Xi_{+}$and $y \in Y$ one has

$$
\xi \cdot y \notin \mathbb{R}^{\times} .
$$

More precisely, for all $0<c<\frac{\pi}{2}$ there exists a $C>0$ such that

$$
(\forall y \in Y)\left(\forall \xi \in \Xi_{c}\right) \quad|1-\xi \cdot y|>C .
$$

Proof. By $G$-equivariance of the form we may assume that $\xi=e^{i t} \xi_{o}$. Let $y=i \mathbf{y}$ for $\mathbf{y} \in \mathbb{R}^{n+1}$. Thus $\xi \cdot y=i e^{i t} \xi_{o} \cdot \mathbf{y}$ with $\xi_{o} \cdot \mathbf{y} \in \mathbb{R}$. As $|t|<\frac{\pi}{2}$, the assertions follow.

We now define the Cauchy-kernel function

$$
\mathcal{K}: \Xi_{+} \times Y \rightarrow \mathbb{C}, \quad(\xi, y) \mapsto \frac{1}{1-\xi \cdot y}
$$

In view of the previous lemma, this function is defined, continuous and bounded on all subsets $\Xi_{c} \times Y$ for $c<\frac{\pi}{2}$. Moreover, $\mathcal{K}$ is a $C R$-function in the first variable and $G$-invariant, i.e., $\mathcal{K}(g \cdot \xi, g \cdot y)=\mathcal{K}(\xi, y)$. In particular, the function

$$
G \ni g \mapsto \mathcal{K}(g)=\mathcal{K}\left(\xi_{o}, g \cdot \mathbf{y}_{0}\right) \in \mathbb{C}
$$


is left $N$-invariant and right $H$-invariant, a fact that we will use in a moment. We will therefore identify $\mathcal{K}$ with a function on $Y$ without further comments. A simple calculation shows that

$$
\mathcal{K}(g)=\frac{1}{1-i\left(g_{0 n}-g_{n n}\right)}
$$

We have $\mathcal{W}_{H}=\{\mathbf{1}\}$, and $\mathcal{W}=\{\mathbf{1}, \epsilon\}$ where $\epsilon=-1$ on $\mathfrak{a}$. As $\epsilon$ corresponds to the matrix $w=\left(\begin{array}{cc}I_{n-1} & 0 \\ 0 & -I_{2}\end{array}\right)$ it follows that

$$
\mathcal{K}\left(a_{z}\right)=\frac{1}{1-i e^{-z}} \quad \text { and } \quad \mathcal{K}\left(a_{z} w\right)=\frac{1}{1+i e^{-z}}
$$

Write $\mathcal{S}(Y)$ for the Schwartz-space on $Y$. Henceforth we will make the assumption that $n$ is even and define the Cauchy - transform by

$$
\mathcal{C}: \mathcal{S}(Y) \rightarrow C R\left(\Xi_{+}\right), \quad \mathcal{C}(f)(\xi)=\int_{Y} \frac{f(y)}{1-\xi \cdot y} d y=\int_{Y} f(y) K(\xi, y) d y
$$

where $d y$ denotes a (suitably normalized) $G$-invariant measure on $Y$.

Theorem 4.2. Let $G=\mathrm{SO}(n, 1)$ with $n=2 k$ even. Let $f \in \mathcal{H}^{2}(D)_{00}$. Then, up to normalization of measures, the Cauchy and the Radon transform coincide, i.e.

$$
\mathcal{C}(f)(\xi)=(-1)^{k-1} 2 \pi \cdot \mathcal{R}(f)(\xi) \quad\left(\xi \in \Xi_{+}\right)
$$

Proof. Since both $\mathcal{C}(f)$ and $\mathcal{R}(f)$ are $C R$-functions, it is sufficient to show that both coincide on $G / M N \subset \Xi$. Moreover, by $G$-equivariance of both maps, it is in fact sufficient to show that

$$
\mathcal{C}(f)\left(\xi_{o}\right)=2 \pi \cdot \mathcal{R}(f)\left(\xi_{o}\right)
$$

Using (4.2) and that $z_{H}^{2 \rho}=(-1)^{k-1} i$ and $z_{H}^{-2 \rho}=\overline{z_{H}^{2 \rho}}=(-1)^{k} i$ we get: 


$$
\begin{aligned}
& \mathcal{C}(f)\left(\xi_{o}\right)=\int_{Y} f(y) \cdot \mathcal{K}(y) d y \\
& =\sum_{w \in \mathcal{W}} \int_{A} \int_{N} f\left(\text { anw } \cdot \mathbf{y}_{o}\right) \cdot \mathcal{K}(\text { anw }) d n d a \\
& =\sum_{w \in \mathcal{W}} \int_{A} \int_{N} f\left(a n w \cdot \mathbf{y}_{o}\right) \cdot \mathcal{K}(a w) d n d a \\
& =\sum_{w \in \mathcal{W}} \int_{A} \int_{N} f\left(a n w z_{H} \cdot \mathbf{x}_{o}\right) \cdot \mathcal{K}(a w) d n d a \\
& =\sum_{w \in \mathcal{W}} \int_{A} \int_{N} f\left(a n z_{H}^{w} \cdot \mathbf{x}_{o}\right) \cdot \mathcal{K}(a w) d n d a \\
& =\sum_{w \in \mathcal{W}} \int_{A} \int_{N} f\left(a n z_{H}^{w} \cdot \mathbf{x}_{o}\right) \cdot\left(z_{H}^{w}\right)^{-2 \rho} \cdot\left(z_{H}^{w}\right)^{2 \rho} \cdot \mathcal{K}(a w) d n d a \\
& =\sum_{w \in \mathcal{W}} \int_{A} \mathcal{R}(f)\left(a z_{H}^{w} \cdot \xi_{o}\right) \cdot\left(z_{H}^{w}\right)^{2 \rho} \cdot \mathcal{K}(a w) d a \\
& =(-1)^{k-1} i\left(\int_{\mathbb{R}} \frac{\mathcal{R}(f)\left(a_{t+i \frac{\pi}{2}} \cdot \xi_{o}\right)}{1-e^{-\left(t+i \frac{\pi}{2}\right)}} d t-\int_{\mathbb{R}} \frac{\mathcal{R}(f)\left(a_{t-i \frac{\pi}{2}} \cdot \xi_{o}\right)}{1-e^{-\left(t-i \frac{\pi}{2}\right)}} d t\right) .
\end{aligned}
$$

Consider the strip domain $S=\left\{z \in \mathbb{C}|| \operatorname{Im} z \mid \leq \frac{\pi}{2}\right\}$. By our assumption on $f$, the function

$$
S \ni z \mapsto F(z)=\frac{i \mathcal{R}(f)\left(a_{z} \cdot \xi_{o}\right)}{1-e^{-z}} \in \mathbb{C}
$$

defines a meromorphic function $F$ on $S$ with a simple pole at $z=0$. Thus the Residue theorem yields that

$$
\mathcal{C}(f)\left(\xi_{o}\right)=(-1)^{k} 2 \pi i \cdot \operatorname{Res}(F, 0)=(-1)^{k-1} 2 \pi \cdot \mathcal{R}(f)\left(\xi_{o}\right)
$$

and this concludes the proof of our theorem.

Remark 4.3. (a) We mention that the geometric pairing $\xi \cdot y$ can be expressed using the previously defined power functions $f_{j}$ (cf. [1.9):

$$
\xi \cdot y=f_{1}\left(\xi^{-1} y\right) \text {. }
$$

(b) The assumption that $n$ is even is not a real restriction as one can slightly modify $\mathcal{C}$ so that it works for all parities (see our discussion in the next section).

\section{The holomorphic Radon transform as Cauchy integral II: Cayley type SPACES}

The example of the hyperboloid discussed in the previous section admits a generalization to NCC spaces of Cayley type. Let us recall that Cayley type spaces are those which are 
associated to Euclidean Jordan algebras $V$ : i.e. $X$ is a tube domain associated to $V$ and $H$ is the structure group of the cone of squares in $V$. In terms of the set of restricted roots $\Sigma$, this means that $\Sigma$ is of type $C_{n}$, say

$$
\Sigma=\left\{\frac{1}{2}\left( \pm \gamma_{i} \pm \gamma_{j}\right) \mid 1 \leq i, j \leq n\right\} \backslash\{0\} .
$$

We assume now that $Y=G / H$ is of Cayley type. Define $T_{j} \in \mathfrak{a}$ by $\gamma_{i}\left(T_{j}\right)=\delta_{i j}$, then

$$
\left.\Omega_{H}=\bigoplus_{j=1}^{n}\right]-\frac{\pi}{2}, \frac{\pi}{2}\left[T_{j} .\right.
$$

As a basis of $\Sigma$ we shall choose

$$
\Pi=\left\{\frac{1}{2}\left(\gamma_{1}-\gamma_{2}\right), \ldots, \frac{1}{2}\left(\gamma_{n-1}-\gamma_{n}\right), \gamma_{n}\right\} .
$$

Obviously $\omega_{1}=-\gamma_{1}$ is a fundamental spherical lowest weight and accordingly $f_{1}(g)=$ $\left\langle\pi_{1}(g) \eta_{j}, v_{1}\right\rangle$ defines a holomorphic function on $G_{\mathbb{C}}$ with $f_{1}(g)=a(g)^{-\gamma_{1}}$ for $g \in N_{\mathbb{C}} A_{\mathbb{C}} K_{\mathbb{C}}$. The analogue of Lemma 4.1 now reads as follows.

Lemma 5.1. Let the natation be as above. Then the following holds:

(1) $f_{1}\left(\exp \left(i \Omega_{H}\right) G z_{H}\right) \subseteq \mathbb{C} \backslash \mathbb{R}^{\times}$.

(2) For all $0 \leq c<1$ there exists $C>0$ such that

$$
(\forall g \in G)\left(\forall Z \in \Omega_{H}\right) \quad\left|1-f_{1}\left(\exp (i c Z) g z_{H}\right)\right|>C .
$$

Proof. First it is clear from (5.1) that

$$
\exp \left(i \Omega_{H}\right)^{\omega_{1}}=\{z \in \mathbb{C} \mid \operatorname{Re} z>0\} .
$$

Next recall that $\bigcup_{w \in \mathcal{W}} N A w H$ is dense in $G$ and that

$$
f_{1}(\text { nawh })=a^{\omega_{1}} z_{H}^{w \omega_{1}} \in \mathbb{R}^{+}\{-i, i\} .
$$

Combine (5.2) and (5.3) and the assertions follow.

For $0<c<1$ define a $G$-subdomains of $\Xi_{+}$by

$$
\Xi_{c}=G \exp \left(i c \Omega_{H}\right) \cdot \xi_{o} .
$$

Note that $-\gamma_{j}, j \neq 1$ is not a fundamental spherical lowest weight. Therefore, for $1 \leq j \leq n$ we define a meromorphic function on $G_{\mathbb{C}}$ directly by

$$
h_{j}(g)=a(g)^{-\gamma_{j}} \quad \text { for } g \in N_{\mathbb{C}} A_{\mathbb{C}} K_{\mathbb{C}} .
$$

Note that $h_{1}=f_{1}$ and, in the same manner as in Lemma 5.1, one establishes that

$$
h_{j}\left(\exp \left(i \Omega_{H}\right) G z_{H}\right) \subseteq \mathbb{C} \backslash \mathbb{R}^{\times} \cup\{\infty\}
$$


In particular we see that the prescription

$$
\mathcal{K}: \Xi_{+} \times Y \rightarrow \mathbb{C}, \quad(\xi, y) \mapsto \prod_{j=1}^{n} \frac{1}{1-h_{j}\left(\xi^{-1} y\right)}
$$

defines an analytic function, $C R$ in the first variable and bounded on all subsets $\Xi_{c} \times Y$.

Remark 5.2. Alternatively, the kernel $\mathcal{K}$ can be expressed in Jordan algebra terms. Let $V$ be the Euclidean Jordan algebra associated to $X$ and $W \subset V$ its cone of squares. Form the tube domains $\mathcal{T}^{ \pm}=V \pm i W \subset V_{\mathbb{C}}$. Then $X=\mathcal{T}^{+}$and $D \simeq \mathcal{T}^{+} \times \mathcal{T}^{-}$with $X$ realized in $D$ via the map $x \mapsto(x, \bar{x})$. Write $\Delta_{j}$ for the power functions on $V_{\mathbb{C}}$ (i.e. generalized principal minors). Then, on $D$, one has

$$
h_{j}(z, w)=\frac{\Delta_{j}(z-w)}{\Delta_{j-1}(z-w)} \quad \text { for }(z, w) \in D
$$

with the understanding that $\Delta_{0} \equiv 1$ Thus $\mathcal{K}$, when considered as a function on $D$, is given by

$$
\mathcal{K}(z, w)=\frac{\Delta_{1}(z-w) \cdot \ldots \cdot \Delta_{n-1}(z-w)}{\prod_{j=1}^{n}\left(\Delta_{j-1}(z-w)-\Delta_{j}(z-w)\right)} .
$$

However, $\mathcal{K}$ is not our Cauchy-kernel yet and some small modification is needed. For that recall the decomposition $\Xi_{+} \simeq G / M N \times \exp \left(i \Omega_{H}\right)$. For a unitary character $\chi \in \widehat{T / F}$, where $F=K \cap T$ is the canonical finite 2-group as usually, define the space of $\chi$-twisted $C R$-functions by

$$
C R_{\chi}\left(\Xi_{+}\right)=\left\{f\left(g a \cdot \xi_{o}\right)=h\left(g a \cdot \xi_{o}\right) \chi^{-1}(a) \mid h \in C R\left(\Xi_{+}\right)\right\} .
$$

Let $\gamma_{0}=\gamma_{1}+\ldots+\gamma_{n}$. In the sequel we will fix $\chi$ to be

$$
\chi=-2 \rho+\gamma_{0}
$$

and notice that $\chi=\mathbf{1}$ for $G=\operatorname{Sl}(2, \mathbb{R})$. For $g \in N_{\mathbb{C}} A T K_{\mathbb{C}}$ write $t(g) \in T / F$ for the compact middle part of $g$ and define the Cauchy-kernel $\mathcal{K}$ by

$$
\mathcal{K}_{\chi}(\xi, y)=\mathcal{K}(\xi, y) \chi\left(t\left(\xi^{-1} y\right)\right) .
$$

and notice that $\mathcal{K}_{\chi}$ is defined whenever $\xi^{-1} y \in N_{\mathbb{C}} A_{\mathbb{C}} K_{\mathbb{C}}$ and, when defined, is in $C R_{\chi}$ as a function of the first variable. We note that $\mathcal{H}_{\chi}$ is $G$-invariant and hence corresponds to a function of one variable $\mathcal{K}_{\chi}(g)=\mathcal{K}_{\chi}\left(\xi_{0}, g \cdot y_{0}\right)$. As before, $\mathcal{K}_{\chi}$ is $N \times H$-invariant, and will be identified with left $N$-invariant function on $Y$.

Whenever defined, we define the twisted Cauchy transform by

$$
\mathcal{C}_{\chi}: \mathcal{S}(Y) \rightarrow C R_{\chi}\left(\Xi_{+}\right), \quad \mathcal{C}_{\chi}(f)(\xi)=\int_{Y} f(y) \mathcal{K}_{\chi}(\xi, y) d y
$$

and the twisted holomorphic Radon transform by

$$
\mathcal{R}_{\chi}: \mathcal{H}^{2}(D)_{00} \rightarrow C R_{\chi}\left(\Xi_{+}\right), \quad f \mapsto\left(\xi=g a \cdot \xi_{o} \mapsto a^{-\gamma_{0}} \int_{N} f\left(g n a \cdot x_{o}\right) d n\right) .
$$


We come to the main result of this section.

Theorem 5.3. Suppose that $Y=G / H$ is of Cayley type. Let $f \in \mathcal{H}^{2}(D)_{00}$. Then, up to normalization of measures, the twisted Cauchy and the twisted Radon transform coincide, i.e.

$$
\mathcal{C}_{\chi}(f)(\xi)=(2 \pi)^{n} \cdot \mathcal{R}_{\chi}(f)(\xi) \quad\left(\xi \in \Xi_{+}\right) .
$$

Proof. Both $\mathcal{C}_{\chi}(f)$ and $\mathcal{R}_{\chi}(f)$ are $C R$-functions, and so it is sufficient to show that both coincide on $G / M N \subset \Xi$. Next, by $G$-equivariance of both maps, it is enough to show that

$$
\mathcal{C}_{\chi}(f)\left(\xi_{o}\right)=(2 \pi)^{n} \cdot \mathcal{R}_{\chi}(f)\left(\xi_{o}\right) .
$$

We now get for the left hand side:

$$
\begin{aligned}
\mathcal{C}_{\chi}(f)\left(\xi_{o}\right) & =\int_{Y} f(y) \cdot \mathcal{K}_{\chi}(y) d y \\
& =\sum_{w \in \mathcal{W} / \mathcal{W}_{H}} \int_{A} \int_{N} f\left(a n w \cdot y_{o}\right) \cdot \mathcal{K}_{\chi}(a n w) d n d a \\
& =\sum_{w \in \mathcal{W} / \mathcal{W}_{H}} \int_{A} \int_{N} f\left(a n w \cdot y_{o}\right) \cdot \mathcal{K}_{\chi}(a w) d n d a \\
& =\sum_{w \in \mathcal{W} / \mathcal{W}_{H}} \int_{A} \int_{N} f\left(a n w z_{H} \cdot x_{o}\right) \cdot \mathcal{K}_{\chi}(a w) d n d a \\
& =\sum_{w \in \mathcal{W} / \mathcal{W}_{H}} \int_{A} \int_{N} f\left(a n z_{H}^{w} \cdot x_{o}\right) \cdot \mathcal{K}_{\chi}(a w) d n d a \\
& =\sum_{w \in \mathcal{W} / \mathcal{W}_{H}} \int_{A} \int_{N} f\left(a n z_{H}^{w} \cdot x_{o}\right) \cdot\left(z_{H}^{w}\right)^{-\gamma_{0}} \cdot\left(z_{H}^{w}\right)^{\gamma_{0}} \cdot \mathcal{K}_{\chi}(a w) d n d a \\
& =\sum_{w \in \mathcal{W} / \mathcal{W}_{H}} \int_{A} \mathcal{R}_{\chi}(f)\left(a z_{H}^{w} \cdot \xi_{o}\right) \cdot\left(z_{H}^{w}\right)^{\gamma_{0}} \cdot \mathcal{K}_{\chi}\left(a z_{H}^{w} \cdot x_{o}\right) d a \\
& \sum_{w \in \mathcal{W} / \mathcal{W}_{H}} \int_{A} \mathcal{R}\left(a z_{H}^{w} \cdot \xi_{o}\right) \cdot\left(z_{H}^{w}\right)^{\gamma_{0}} \cdot \mathcal{K}\left(a z_{H}^{w} \cdot x_{o}\right) d a .
\end{aligned}
$$

Specifically we have $\mathcal{W} / \mathcal{W}_{H} \simeq\left(\mathbb{Z}_{2}\right)^{n}$ and $z_{H}^{w \gamma_{0}}=i^{n} \operatorname{sgn}(w)$

$$
\begin{aligned}
& \mathcal{C}_{\chi}(f)\left(\xi_{o}\right)=i^{n} \sum_{\varepsilon \in\left(\mathbb{Z}_{2}\right)^{n}} \operatorname{sgn}(\varepsilon) \int_{\mathbb{R}^{n}} \mathcal{R}(f)\left(\exp \left(\sum_{j=1}^{n}\left(t_{j}+i \varepsilon_{j} \frac{\pi}{2}\right) T_{j}\right) \cdot \xi_{o}\right) \cdot \\
& \cdot \mathcal{K}\left(\exp \left(\sum_{j=1}^{n}\left(t_{j}+i \varepsilon_{j} \frac{\pi}{2}\right) T_{j}\right) \cdot x_{o}\right) d t
\end{aligned}
$$


Next observe that

$$
\mathcal{K}\left(\exp \left(\sum_{j=1}^{n}\left(t_{j}+i \varepsilon_{j} \frac{\pi}{2}\right) T_{j}\right) \cdot x_{o}\right)=\prod_{j=1}^{n} \frac{1}{1-e^{-\left(t_{j}+i \varepsilon_{j} \frac{\pi}{2}\right)}} .
$$

Let us consider the multi strip domain $S=\left\{z \in \mathbb{C}^{n}|| \operatorname{Im} z_{j} \mid \leq \frac{\pi}{2}\right\}$. By our assumption on $f$, the prescription

$$
S \ni z \mapsto \mathcal{R}(f)\left(\exp \left(\sum_{j=1}^{n} z_{j} T_{j}\right) \cdot \xi_{o}\right) \cdot \prod_{j=1}^{n} \frac{1}{1-e^{-z_{j}}} \in \mathbb{C}
$$

defines a meromorphic function $F$ on $S$ with simple multi-pole at $z_{j}=0$. Thus iteratively applying the the Residue theorem yields that

$$
\mathcal{C}_{\chi}(f)\left(\xi_{o}\right)=(-2 \pi i)^{n} \cdot \operatorname{Res}(F, 0)=(2 \pi)^{n} \cdot \mathcal{R}(f)\left(\xi_{o}\right)=(2 \pi)^{n} \mathcal{R}_{\chi}(f)\left(\xi_{o}\right)
$$

and it concludes the proof of our theorem.

\section{SOME REMARKS ON THE INVERSION OF THE HOLOMORPHIC RADON TRANSFORM}

The inversion of the real horospherical transform on $X$ can be analytically continued to give the inversion of the holomorphic horospherical transform. The dual transform is given by integration over the real form $S_{\mathbb{R}}^{K}$ of $S(z)$. However, there is a second non-compact real form $S_{\mathbb{R}}^{H}(z)$ of $S(z)$ which gives rise to a different dual transform and inversion. This is the topic of this section. Mainly we will focus on $G=\operatorname{Sl}(2, \mathbb{R})$.

We begin with the definition of an appropriate function space. Let us denote by $\mathcal{F}\left(\Xi_{+}\right)$ denote the space of $C R$-functions on $\Xi_{+}$which extend continuously to $G \exp \left(i \bar{\Omega}_{H}\right) \cdot \xi_{o}$ such that $H \ni h \mapsto f\left(g h z_{H} \cdot \xi_{o}\right) \in \mathbb{C}$ is integrable for all $g \in G$. For those functions we define the dual Radon transform by

$$
\mathcal{F}\left(\Xi_{+}\right) \rightarrow C(Y), \quad \phi \mapsto \phi^{\vee}
$$

with

$$
\phi^{\vee}(y)=\int_{H} f\left(g h z_{H} \cdot \xi_{o}\right) d h=\int_{S_{\mathbb{R}}(y)} f \quad\left(y=g \cdot y_{o} \in Y\right) .
$$

Clearly, this is a $G$-equivariant mapping.

We would like to understand the relation between $\mathcal{R}$ and $\phi \mapsto \phi^{\vee}$. In this context we would like to mention the result in [10] for the holomorphic discrete series: there exists a differential operator $\mathcal{L}$ such that $(\mathcal{L} \mathcal{R}(f))^{\vee}=f$. Hence it is natural to ask whether a similar statement would hold true for the most continuous spectrum considered in this paper. It will turn out that the situation different for the most continuous series in the sense that the inverting operator $\mathcal{L}$ is not a differential operator. We give a detailed discussion for the the basic example. 
6.1. The example of $G=\operatorname{Sl}(2, \mathbb{R})$. For this paragraph we let $G=\operatorname{Sl}(2, \mathbb{R})$ with the usual choices

$$
A=\left\{\left(\begin{array}{cc}
t & 0 \\
0 & \frac{1}{t}
\end{array}\right) \mid t>0\right\}, \quad N=\left\{\left(\begin{array}{ll}
1 & x \\
0 & 1
\end{array}\right) \mid x \in \mathbb{R}\right\}
$$

and $K=\mathrm{SO}(2, \mathbb{R})$. Let $f \in \mathcal{H}^{2}(D)_{00}$ be a $K$-invariant function. In the sequel we will identify $\mathfrak{a}_{\mathbb{C}}^{*}$ with $\mathbb{C}$ via the assignment

$$
\mathbb{C} \ni \lambda \mapsto \lambda \cdot \rho \in \mathfrak{a}_{\mathbb{C}}^{*}
$$

In this coordinates one has

$$
\mathbf{c}(\lambda)=\pi^{-1 / 2} \frac{\Gamma(\lambda / 2)}{\Gamma((\lambda+1) / 2)} \quad \text { and } \quad|\mathbf{c}(\lambda)|^{-2}=\frac{i \pi \lambda}{2} \tanh \left(\frac{i \pi \lambda}{2}\right)
$$

We know that $\left.f\right|_{X} \in L^{2}(X)$ and, as $f$ is $K$-invariant, we can write

$$
f(x)=\frac{1}{2} \int_{\mathbb{R}} \hat{f}(i \lambda) \phi_{i \lambda}(x) \frac{d \lambda}{|\mathbf{c}(i \lambda)|^{2}} \quad(x \in X) .
$$

Applying $\mathcal{R}$ yields that

$$
\mathcal{R}(f)(\xi)=\frac{1}{2} \int_{\mathbb{R}} \hat{f}(i \lambda) a\left(\xi^{-1}\right)^{\rho(1+i \lambda)} d \lambda
$$

and thus

$$
\mathcal{R}(f)^{\vee}\left(y_{o}\right)=\frac{1}{2} \int_{H} \int_{\mathbb{R}} \hat{f}(i \lambda) a\left(z_{H}^{-1} h\right)^{\rho(1+i \lambda)} d \lambda d h .
$$

Now, for $h=\left(\begin{array}{cc}\cosh t & \sinh t \\ \sinh t & \cosh t\end{array}\right) \in H=\mathrm{SO}_{e}(1,1)$ one has $z_{H}^{-1} h=\left(\begin{array}{cc}e^{-i \frac{\pi}{4}} \cosh t & e^{-i \frac{\pi}{4}} \sinh t \\ e^{i \frac{\pi}{4}} \sinh t & e^{i \frac{\pi}{4}} \cosh t\end{array}\right)$ and so

$$
a\left(z_{H}^{-1} h\right)^{\rho}=\left(\frac{1}{i\left(\sinh ^{2} t+\cosh ^{2} t\right)}\right)^{\frac{1}{2}}=e^{-i \frac{\pi}{4}} \cdot \frac{1}{(\cosh 2 t)^{\frac{1}{2}}}
$$

Therefore we obtain that

$$
\mathcal{R}(f)^{\vee}\left(y_{o}\right)=\frac{1}{2} \int_{\mathbb{R}} \int_{\mathbb{R}} \hat{f}(i \lambda) \cdot \frac{e^{\frac{\pi}{4}(\lambda-i)}}{(\cosh 2 t)^{\frac{1}{2}(1+i \lambda)}} d \lambda d t .
$$

\section{Lemma 6.1.}

$$
\int_{\mathbb{R}} \frac{1}{(\cosh 2 t)^{\frac{1}{2}(1+i \lambda)}} d t=\frac{1}{2} B(1 / 2,(1+i \lambda) / 4)=\frac{1}{2} \frac{\Gamma((1+i \lambda) / 4) \Gamma(1 / 2)}{\Gamma((3+i \lambda) / 4)}
$$


Proof. Let us denote the integral on the left hand side by $I(\lambda)$. With the substitution $u=\cosh 2 t$ we obtain

$$
\begin{aligned}
I(\lambda) & =\int_{1}^{\infty} \frac{1}{u^{\frac{1}{2}(1+i \lambda)}} \frac{1}{\left(u^{2}-1\right)^{1 / 2}} d u \\
& =\frac{1}{2} \int_{1}^{\infty} v^{-\frac{1+i \lambda}{4}}(v-1)^{-1 / 2} v^{-1 / 2} d v \quad\left(v=u^{2}\right) \\
& =\frac{1}{2} B(1 / 2,(1+i \lambda) / 4)
\end{aligned}
$$

as $B(p, q)=\int_{1}^{\infty} u^{-(p+q)}(u-1)^{p-1} d u$.

Using this, we get:

$$
\mathcal{R}(f)^{\vee}\left(y_{o}\right)=\frac{1}{4} \int_{\mathbb{R}} \hat{f}(i \lambda) \cdot e^{\frac{\pi}{4}(\lambda-i)} B(1 / 2,(1+i \lambda) / 4) d \lambda .
$$

Define

$$
C_{1}(\lambda)=e^{\frac{\pi}{4}(\lambda-i)} B(1 / 2,(1+i \lambda) / 4)+e^{-\frac{\pi}{4}(\lambda+i)} B(1 / 2,(1+i \lambda) / 4)
$$

and note that $\lambda \mapsto \hat{f}(i \lambda)$ is an even function. Thus (6.2) yields that

$$
\mathcal{R}(f)^{\vee}\left(y_{o}\right)=\frac{1}{2} \int_{\mathbb{R}} \hat{f}(i \lambda) \cdot C_{1}(\lambda) d \lambda .
$$

By the Fourier inversion formula, we have

$$
f\left(y_{o}\right)=\frac{1}{2} \int_{\mathbb{R}} \hat{f}(i \lambda) \phi_{i \lambda}\left(y_{o}\right) \frac{d \lambda}{|\mathbf{c}(\lambda)|^{2}} .
$$

Now, from the special values of the Gauß hypergeometric function we get

$$
\phi_{i \lambda}\left(y_{o}\right)=F(1 / 4+i \lambda / 4,1 / 4-i \lambda / 4,1 ; 1)=\frac{1}{2 B((3-i \lambda) / 4,(3+i \lambda) / 4)} .
$$

We therefore define

$$
C_{2}(\lambda)=\frac{1 / 2}{B((3-i \lambda) / 4,(3+i \lambda) / 4)|\mathbf{c}(i \lambda)|^{2}}
$$

and note that (6.4) transforms into

$$
f\left(y_{o}\right)=\frac{1}{2} \int_{\mathbb{R}} \hat{f}(i \lambda) \cdot C_{2}(\lambda) d \lambda
$$

In the next step we want to compare the expressions $C_{1}(\lambda)$ and $C_{2}(\lambda)$. If there would exist a differential operator $\mathcal{L}$, then there should be a polynomial relation between $C_{1}$ and $C_{2}$.

We first consider

$$
c_{1}(\lambda):=C_{1}(\lambda) \cdot\left(\frac{\Gamma(1 / 2)}{\Gamma(3 / 4-i \lambda / 4) \Gamma(3 / 4+i \lambda / 4)}\right)^{-1}=C_{1}^{+}(\lambda)+C_{1}^{-}(\lambda)
$$


with

$$
C_{1}^{ \pm}(\lambda)=e^{ \pm \frac{\pi \lambda}{4}-i \frac{\pi}{4}} \cdot \frac{\Gamma((1 \pm i \lambda) / 4) \Gamma(1 / 2)}{\Gamma(3 / 4 \pm i \lambda / 4)} \cdot\left(\frac{\Gamma(1 / 2)}{\Gamma(3 / 4-i \lambda / 4) \Gamma(3 / 4+i \lambda / 4)}\right)^{-1}
$$

We focus on $C_{1}^{+}$and obtain

$$
\begin{aligned}
C_{1}^{+}(\lambda) & =e^{\frac{\pi}{4}(\lambda-i)} \cdot \frac{\Gamma((1+i \lambda) / 4) \Gamma(1 / 2)}{\Gamma(3 / 4+i \lambda / 4)} \cdot\left(\frac{\Gamma(1 / 2)}{\Gamma(3 / 4-i \lambda / 4) \Gamma(3 / 4+i \lambda / 4)}\right)^{-1} \\
& =e^{\frac{\pi}{4}(\lambda-i)} \cdot \Gamma((1+i \lambda) / 4) \Gamma(3 / 4-i \lambda / 4) \\
& =e^{\frac{\pi}{4}(\lambda-i)} \cdot \Gamma((1+i \lambda) / 4) \Gamma(1+(-1 / 4-i \lambda / 4)) \\
& =e^{\frac{\pi}{4}(\lambda-i)} \cdot(-1 / 4-i \lambda / 4) \Gamma((1+i \lambda) / 4) \Gamma(-(1+i \lambda) / 4) \\
& =\frac{e^{\frac{\pi}{4}(\lambda-i)} \pi}{\sin \pi(1 / 4+i \lambda / 4)}
\end{aligned}
$$

Likewise we obtain

$$
C_{1}^{-}(\lambda)=C_{1}^{+}(-\lambda)=\frac{e^{-\frac{\pi}{4}(\lambda+i)} \pi}{\sin \pi(1 / 4-i \lambda / 4)}
$$

and so

$$
\begin{aligned}
c_{1}(\lambda) & =\frac{e^{\frac{\pi}{4}(\lambda-i)} \pi}{\sin \pi(1 / 4+i \lambda / 4)}+\frac{e^{-\frac{\pi}{4}(\lambda+i)} \pi}{\sin \pi(1 / 4-i \lambda / 4)} \\
& =\frac{\pi}{i} \frac{e^{\frac{\pi}{4}(\lambda-i)}}{\sinh \pi(\lambda / 4-i / 4)}+\frac{\pi}{i} \frac{e^{-\frac{\pi}{4}(\lambda+i)}}{\sinh \pi(-\lambda / 4-i / 4)} \\
& =\frac{\pi}{i} \frac{\cosh \left(\pi \lambda 4-i \frac{\pi}{4}\right)+\sinh \left(\frac{\pi \lambda}{4}-i \frac{\pi}{4}\right)}{\sinh \pi(\lambda / 4-i / 4)}+\frac{\pi}{i} \frac{\cosh \left(-\frac{\pi \lambda}{4}-i \frac{\pi}{4}\right)+\sinh \left(-\frac{\pi \lambda}{4}-i \frac{\pi}{4}\right)}{\sinh \pi(-\lambda / 4-i / 4)} \\
& =\frac{\pi}{i} \cdot\left(2+\operatorname{coth}\left(\frac{\pi}{4}(\lambda-i)\right)+\operatorname{coth}\left(-\frac{\pi}{4}(\lambda+i)\right)\right)
\end{aligned}
$$

Now define $g(\lambda)$ by the requirement

$$
g(\lambda) c_{1}(\lambda)=\frac{1}{|\mathbf{c}(i \lambda)|^{2}}=\frac{\pi \lambda}{2} \tanh \left(\frac{\pi \lambda}{2}\right)
$$

Now note that 


$$
\begin{aligned}
c_{1}(\lambda+i) & =\frac{\pi}{i} \cdot\left(2+\operatorname{coth}\left(\frac{\pi \lambda}{4}\right)+\operatorname{coth}\left(-\frac{\pi \lambda}{4}-i \frac{\pi}{2}\right)\right) \\
& =\frac{\pi}{i} \cdot\left(2+\operatorname{coth}\left(\frac{\pi \lambda}{4}\right)-\tanh \left(\frac{\pi \lambda}{4}\right)\right) \\
& =\frac{\pi}{i} \cdot\left(2+\frac{2}{\sinh \left(\frac{\pi \lambda}{2}\right)}\right)
\end{aligned}
$$

and thus we get

$$
g(\lambda+i) \frac{\pi}{i} \cdot\left(2+\frac{2}{\sinh \left(\frac{\pi \lambda}{2}\right)}\right)=\frac{\pi(\lambda+i)}{2} \operatorname{coth}\left(\frac{\pi \lambda}{2}\right) .
$$

Further manipulation then yields that

$$
g(\lambda)=\frac{i \lambda}{4} \cdot \frac{\sinh \left(\frac{\pi \lambda}{2}\right)}{1-\cosh \left(\frac{\pi \lambda}{2}\right)}
$$

and it is obvious that $g$ is not a polynomial function. Since

$$
g(\lambda) C_{1}(\lambda)=C_{2}(\lambda)
$$

it is now clear that there exists no differential operator $\mathcal{L}$ which inverts the holomorphic Radon transform. However the function $g(\lambda)$ defines us a spectral multiplier which is a pseudo-differential operator which we now call $\mathcal{L}$. We summarize our discussion.

Theorem 6.2. Let $G=\operatorname{Sl}(2, \mathbb{R})$ and $\mathcal{L}$ the spectral multiplier defined by the function $g(\lambda)=$ $\frac{i \lambda}{4} \cdot \frac{\sinh \left(\frac{\pi \lambda}{2}\right)}{1-\cosh \left(\frac{\pi \lambda}{2}\right)}$. Then for sufficiently decaying functions $f$ on $Y$ we have

$$
f=(\mathcal{L} \mathcal{R}(f))^{\vee} .
$$

\section{Geometric definition of the Hardy space}

This final section deals with the structure of the Hardy space $\mathcal{H}^{2}(D)$. It allows independent reading and is of independent interest.

Initially, the Hardy space was defined spectrally (see 9]). Below we will show how to define the Hardy space geometrically, i.e. we give geometric definition of the norm on $\|\cdot\|_{H}$ on $\mathcal{H}^{2}(D)$ through $G$-orbit integrals on $D$. For that we start by recalling the orbital integral $\mathbf{O}_{h}$ and the pseudo-differential operator $\mathcal{D}$ introduced also used in [15].

7.1. $G$-orbit integrals on the domain $D$. For a sufficiently decaying functions $h$ on $D$ we define its $G$-orbit integral on $D$ as the following function on $i 2 \Omega_{H}$

$$
\mathbf{O}_{h}(i X)=\int_{G} h\left(g \exp \left(i \frac{1}{2} X\right) \cdot x_{o}\right) d g \quad\left(X \in 2 \Omega_{H}\right) .
$$


For $f \in \mathcal{H}^{2}(D)$ we notice that $|f|^{2}$ is a sufficiently decaying function on $D$, i.e. $\mathbf{O}_{|f|^{2}}(i X)$ is finite for all $X \in 2 \Omega_{H}$. Moreover, in view of (3.7) we see that $\mathbf{O}_{|f|^{2}}$ has a natural holomorphic extension to a holomorphic function on the abelian tube domain $\mathcal{T}\left(2 \Omega_{H}\right)=\mathfrak{a}+i 2 \Omega_{H}$, namely

$$
\mathbf{O}_{|f|^{2}}(Z)=\int_{\mathcal{X}}|\hat{f}(b, \lambda)|^{2} \varphi_{\lambda}(\exp (Z)) d \mu_{\mathcal{X}}(b, \lambda) \quad\left(Z \in \mathcal{T}\left(2 \Omega_{H}\right)\right)
$$

7.2. A certain pseudo-differential shift operator. Define a space $\mathcal{F}\left(\mathcal{T}\left(2 \Omega_{H}\right)\right)$ of $\mathcal{W}$ invariant holomorphic functions on the tube domain $\mathcal{T}\left(2 \Omega_{H}\right)$ by the following property: $f \in \mathcal{F}\left(\mathcal{T}\left(2 \Omega_{H}\right)\right)$ if $f$ can be written as

$$
f(Z)=\int_{i \mathfrak{a}_{+}^{*}} h(\lambda) \varphi_{\lambda}(\exp (Z)) \frac{d \lambda}{|\mathbf{c}(\lambda)|^{2}} \quad\left(Z \in \mathcal{T}\left(2 \Omega_{H}\right)\right)
$$

where $h \in L^{1}\left(i \mathfrak{a}_{+}^{*}, \frac{\cosh (\lambda)}{|\mathbf{c}(\lambda)|^{2}} d \lambda\right)$. If $Q \subset \mathcal{T}\left(2 \Omega_{H}\right)$ is compact, then there exists a constant $C_{Q}>0$ such that

$$
\left(\forall \lambda \in i \mathfrak{a}^{*}\right) \quad \sup _{X \in Q}\left|\varphi_{\lambda}(\exp (i 2 X))\right| \leq C_{Q} \cosh (\lambda) .
$$

As $\frac{1}{|\mathbf{c}(\lambda)|^{2}}$ is at most of polynomial growth, it follows that $f$ is indeed holomorphic and $\mathcal{W}$ invariant. Moreover, $f$ is uniquely determined by $h$. It follows from our discussion that the prescription

$$
\mathcal{D}: \mathcal{F}\left(\mathcal{T}\left(2 \Omega_{H}\right)\right) \rightarrow \mathcal{O}\left(\mathcal{T}\left(2 \Omega_{H}\right)\right)^{\mathcal{W}} ; \quad(\mathcal{D} F)(Z)=\int_{i \mathfrak{a}_{+}^{*}} h(\lambda) \sum_{w \in \mathcal{W}} e^{\lambda(w Z)} \frac{d \lambda}{|\mathbf{c}(\lambda)|^{2}}
$$

is a well defined linear mapping.

Remark 7.1. The operator $\mathcal{D}$ is a pseudo-differential shift operator and a differential operator if all multiplicities are even. The operator $\mathcal{D}$ is related to the Abel transform as explained in [15], Remark 3.2.

Example 7.2. In this example we discuss the operator $\mathcal{D}$ when the underlying group $G$ is complex. Then $\mathcal{D}$ is a differential operator of a particularly nice form.

If $G$ is complex, then there is an explicit formula for spherical functions, due to HarishChandra:

$$
\varphi_{\lambda}(\exp (Z))=\mathbf{c}(\lambda) \frac{\sum_{w \in \mathcal{W}} \varepsilon(w) e^{\lambda(w Z)}}{\prod_{\alpha \in \Sigma^{+}} 2 \sinh \alpha(Z)}
$$

for all $Z \in \mathcal{T}\left(2 \Omega_{H}\right)$. The c-function has the familiar form

$$
\mathbf{c}(\lambda)=\frac{\prod_{\alpha \in \Sigma^{+}}\langle\rho, \alpha\rangle}{\prod_{\alpha \in \Sigma^{+}}\langle\lambda, \alpha\rangle}
$$

For each $\alpha \in \Sigma$ let $A_{\alpha} \in \mathfrak{a}$ be such that $\alpha=\left\langle\cdot, A_{\alpha}\right\rangle$. Furthermore let $\partial_{\alpha}$ be the partial derivative on $\mathcal{T}\left(2 \Omega_{H}\right)$ in direction $A_{\alpha}$. Define a partial differential operator on $\mathcal{T}\left(2 \Omega_{H}\right)$ by 
$\partial_{\Sigma^{+}}=\prod_{\alpha \in \Sigma^{+}} \partial_{\alpha}$. Finally with $J(Z)=\prod_{\alpha \in \Sigma^{+}} 2 \sinh \alpha(Z)$ we declare a differential operator on $\mathcal{T}\left(2 \Omega_{H}\right)$ by

$$
\mathcal{D}=\text { const } \cdot \partial_{\Sigma^{+}} \circ J
$$

with const $=\prod_{\alpha \in \Sigma^{+}}\langle\rho, \alpha\rangle$. The relation

$$
\mathcal{D}\left(\varphi_{\lambda} \circ \exp \right)(Z)=\sum_{w \in \mathcal{W}} e^{\lambda(w Z)}
$$

is now obvious.

7.3. The geometric norm. For a function $f \in \mathcal{H}^{2}(D)$ let us write $\|f\|_{H}$ for its norm as before. By Lemma 3.2 this norm is given by

$$
\|f\|_{H}=\int_{\mathcal{X}}|\hat{f}(b, \lambda)|^{2} \cosh (\lambda) d \mu_{\mathcal{X}}(b, \lambda)
$$

The objective of this section is to express $\|f\|_{H}$ in terms of the much more geometric orbital integrals $\mathbf{O}_{|f|^{2}}$. Our result is as follows.

Theorem 7.3. Let $f \in \mathcal{H}^{2}(D)$. Then $\mathbf{O}_{|f|^{2}} \in \mathcal{F}\left(\mathcal{T}\left(2 \Omega_{H}\right)\right)$ and the Hardy space norm $\|f\|_{H}$ of $f$ is given by

$$
\|f\|_{H}=\sup _{X \in 2 \Omega_{H}} \frac{\mathcal{D} \mathbf{O}_{|f|^{2}}(i X)}{\left|\mathcal{W}_{H}\right|} .
$$

In particular, the Hardy space $\mathcal{H}^{2}(D)$ can be defined as

$$
\mathcal{H}^{2}(D)=\left\{f \in \mathcal{O}(D) \mid \mathbf{O}_{|f|^{2}} \in \mathcal{F}\left(\mathcal{T}\left(2 \Omega_{H}\right)\right) \sup _{X \in 2 \Omega_{H}} \frac{\mathcal{D} \mathbf{O}_{|f|^{2}}(i X)}{\left|\mathcal{W}_{H}\right|}<\infty\right\} .
$$

Proof. Fix $f \in \mathcal{H}^{2}(D)$. By equation (7.1) we have

$$
\mathbf{O}_{|f|^{2}}(Z)=\int_{\mathcal{X}}|\hat{f}(b, \lambda)|^{2} \varphi_{\lambda}(\exp (Z)) d \mu_{\mathcal{X}}(b, \lambda)
$$

for all $Z \in \mathcal{T}\left(2 \Omega_{H}\right)$. By the spectral definition of $\mathcal{H}^{2}(D)$ it follows that

$$
h(\lambda):=\int_{B}|\hat{f}(b, \lambda)|^{2} d b
$$

defines a function $h \in L^{1}\left(i \mathfrak{a}_{+}^{*}, \frac{\cosh (\lambda)}{|\mathbf{c}(\lambda)|^{2}} d \lambda\right)$. Thus $\mathbf{O}_{|f|^{2}} \in \mathcal{F}\left(\mathcal{T}\left(2 \Omega_{H}\right)\right)$ and the application of $\mathcal{D}$ to $\mathbf{O}_{|f|^{2}}$ yields

$$
\left(\mathcal{D} \mathbf{O}_{|f|^{2}}\right)(Z)=\int_{\mathcal{X}}|\hat{f}(b, \lambda)|^{2} \sum_{w \in \mathcal{W}} e^{\lambda(Z)} d \mu_{\mathcal{X}}(b, \lambda) .
$$

Now notice that

$$
\frac{1}{\left|\mathcal{W}_{H}\right|} \sum_{w \in \mathcal{W}} e^{\lambda(i w X)} \leq \cosh (\lambda) \quad\left(X \in 2 \Omega_{H}\right)
$$


and

$$
\sup _{X \in 2 \Omega_{H}} \sum_{w \in \mathcal{W}} e^{\lambda(i w X)}=\lim _{X \rightarrow Z_{H}} \sum_{w \in \mathcal{W}} e^{\lambda(i 2 w X)}=\left|\mathcal{W}_{H}\right| \cdot \cosh (\lambda) .
$$

The claim follows now from the spectral definition of the norm in $\mathcal{H}^{2}(D)$. Finally, backtracking the steps of the proof readily yields (7.4).

Remark 7.4. Some comments on the geometric Hardy norm

$$
\|f\|_{H}=\sup _{Z \in \Omega_{H}} \frac{1}{\left|\mathcal{W}_{H}\right|} \cdot\left(\mathcal{D} \mathbf{O}_{|f|^{2}}\right)(Z)
$$

seem to be appropriate. Usually, in the theory of Hardy spaces (e.g. Hardy space on the upper half plane) one takes the supremum over a family of $L^{2}$-integrals over totally real submanifolds. In our case one takes a supremum over $G$-orbits, which for the exception of the orbit through the origin, are never totally real. Secondly, we find the appearance of the pseudo differential operator $\mathcal{D}$ interesting. In the context of Hardy spaces it might be novel.

7.4. The $K$-invariant case. In this subsection we give another description of the subspace $\mathcal{H}^{2}(D)^{K}$ using the Abel transform and the results in Appendix A. We start by noting the following simple connection between the Abel transform and the Fourier transform of a $K$-invariant function. For that we note first, that $\hat{f}(b, \lambda)$ is independent of $b \in B$ if $f$ is $K$-invariant. We write then simply $\hat{f}(\lambda)$ and note that $\hat{f}$ is $\mathcal{W}$-invariant. Furthermore

$$
\begin{aligned}
\hat{f}(\lambda) & =\int_{X} f(x) a(x)^{\rho-\lambda} d x \\
& =\int_{A} \int_{N} f\left(n a \cdot x_{o}\right) a^{-\rho-\lambda} d n d a \\
& =\mathcal{F}_{A}(\mathcal{A}(f))(\lambda)
\end{aligned}
$$

where $\mathcal{F}_{A}$ stands for the Fourier transform on the abelian group $A$. Recall, that $\mathcal{F}: L^{2}(A) \rightarrow$ $L^{2}\left(i \mathfrak{a}^{*},|\mathcal{W}|^{-1} d \lambda\right)$ is a unitary isomorphism. Define a multiplication operator $D_{\mathfrak{a}}$ on $i \mathfrak{a}^{*}$ by $D_{\mathfrak{a}}(F)=\mathbf{c}(-\lambda)^{-1} F$ and denote the corresponding multiplier operator on $A$ by $D_{A}$. Let $\Lambda:=D_{A} \circ \mathcal{A}$. Finally, we define a multiplier $m$ on $\mathcal{W} \times i \mathfrak{a}^{*}$ by $m(s, \lambda)=\mathbf{c}(-s \lambda) / \mathbf{c}(-\lambda)$. We denote by $\tau$ the corresponding representation $\tau(s) f(\lambda)=m\left(s^{-1}, \lambda\right) f\left(s^{-1} \lambda\right)$. Then, cf. [19], Section 1, in particular Lemma 1.4, we have a commutative diagram, where each of the maps is an unitary isomorphism:

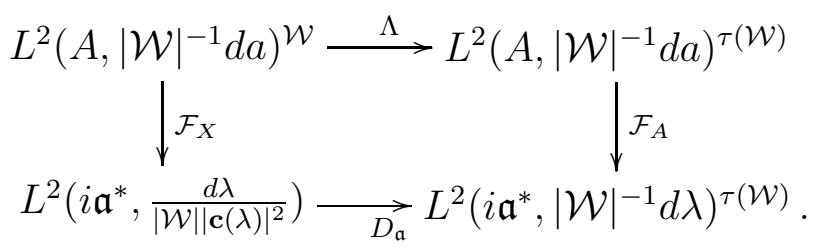

Recall the Hardy space $\mathcal{H}^{2}\left(T\left(\Omega_{H}\right)\right)$ from Appendix A and its spectral description in Theorem [7.9. It follows then from Theorem 3.2, and the obvious renormalization of measures, as 
we have not included the $2 \pi$ in the exponential function, that $\Lambda\left(\mathcal{H}^{2}(D)^{K}\right) \subseteq \mathcal{H}^{2}\left(T\left(\Omega_{H}\right)\right)^{\tau(\mathcal{W})}$. As $\Lambda: L^{2}\left(A,|\mathcal{W}|^{-1} d a\right)^{\mathcal{W}} \rightarrow L^{2}\left(A,|\mathcal{W}|^{-1} d a\right)^{\tau(\mathcal{W})}$ is a unitary isomorphism, we get:

Theorem 7.5. The map $\Lambda: \mathcal{H}^{2}(D)^{K} \rightarrow \mathcal{H}^{2}\left(T\left(\Omega_{H}\right)\right)^{\tau(\mathcal{W})}$ is a unitary isomorphism.

Example 7.6. If $G$ has complex structure, then according to [19], Example 1.12, the map $\Lambda$ is a multiplication operator given by

$$
\Lambda(f)(a)=\left(\prod_{\alpha \in \Sigma^{+}} \sinh \langle\alpha, \log (a)\rangle\right) f(a) .
$$

We now determine the reproducing kernel for $\mathcal{H}^{2}(D)^{K}$. One could easily deduct that from Theorem 7.10 but we will give another proof, that follows similar arguments.

Theorem 7.7. The reproducing kernel $\mathcal{K}(z, w)$ for $\mathcal{H}^{2}(D)^{K}$ is given by

$$
\mathcal{K}(z, w)=\int_{i \mathfrak{a}_{+}^{*}} \varphi_{\lambda}(z) \varphi_{-\lambda}(\bar{w}) d \mu(\lambda) .
$$

Proof. Let $f \in \mathcal{H}^{2}(D)^{K}$ and $w \in D$. Recall, that by Lemma 3.2 we have $\Phi(g)(\lambda)=$ $\hat{g}(\lambda) \cosh (\lambda)$ for all $g \in \mathcal{H}^{2}(D)^{K}$. Therefore

$$
\begin{aligned}
f(w) & =\left\langle f, K_{w}\right\rangle \\
& =\int_{i \mathfrak{a}_{+}} \hat{f}(\lambda) \cosh (\lambda) \overline{\widehat{K_{w}}(\lambda) \cosh (\lambda)} d \mu(\lambda) \\
& =\int_{i \mathfrak{a}_{+}^{*}} \hat{f}(\lambda) \overline{\widehat{K_{w}}(\lambda) \cosh (\lambda)} \frac{d \lambda}{|\mathbf{c}(\lambda)|^{2}} \\
& =\int_{i \mathfrak{a}_{+}^{*}} \hat{f}(\lambda) \varphi_{\lambda}(w) \frac{d \lambda}{|\mathbf{c}(\lambda)|^{2}} .
\end{aligned}
$$

Thus

$$
\widehat{K_{w}}(\lambda)=\frac{\varphi_{-\lambda}(\bar{w})}{\cosh (\lambda)}
$$

From this we now get:

$$
\begin{aligned}
& K(z, w)=\left\langle K_{w}, K_{z}\right\rangle \\
& =\int_{i \mathfrak{a}_{+}^{*}} \widehat{K_{w}}(\lambda) \cosh (\lambda) \overline{\widehat{K}_{z}}(\lambda) \cosh (\lambda) d \mu(\lambda) \\
& =\int_{i \mathfrak{a}_{+}^{*}} \varphi_{-\lambda}(\bar{w}) \varphi_{\lambda}(z) d \mu(\lambda)
\end{aligned}
$$

and the claim follows. 


\section{APPENDIX: HARDY SPACES ON STRIP DOMAIN}

We let $V$ be an Euclidean vector space, e.g. $V=\mathbb{R}^{n}$ endowed with the standard inner product. Denote by $\mathrm{O}(V)$ the orthogonal group of $V$ and let $\mathcal{W} \subset \mathrm{O}(V)$ be a finite subgroup which acts irreducibly on $V$. We fix $y_{o} \in V, y_{o} \neq 0$ and set

$$
\Omega=\operatorname{int}\left(\left\{\text { convex hull of } \mathcal{W}\left(y_{o}\right)\right\}\right) \text {. }
$$

Notice that $\Omega$ is the interior of a compact polyhedron and that $0 \in \Omega$. Write $V_{\mathbb{C}}:=V \otimes_{\mathbb{R}} \mathbb{C} \simeq$ $V+i V$ for the complexification of $V$ and define a tube domain in $V_{\mathbb{C}}$ by

$$
T(\Omega)=V+i \Omega .
$$

Let us denote by $d x$ the measure $(2 \pi)^{-\operatorname{dim} V / 2}$ times the normalized Lebesgue measure on $V$. Then the Fourier transform

$$
f \mapsto \mathcal{F} f(\lambda)=\int_{V} f(x) e^{-\langle\lambda, x\rangle} d x
$$

is a unitary $L^{2}$-isomorphism. $V^{*}$ is the dual of $V$, and $\langle\lambda \cdot x\rangle=\lambda(x)$ denotes the standard duality between $V$ and $V^{*}$. Denote by $\mathcal{O}(T(\Omega))$ the space of holomorphic functions on $T(\Omega)$. The Hardy space $\mathcal{H}^{2}(T(\Omega)$ is defined by:

$$
\mathcal{H}^{2}(T(\Omega)):=\left\{\left.f \in \mathcal{O}(T(\Omega))\left|\|f\|_{\mathcal{H}}^{2}=\sup _{y \in \Omega} \int_{V}\right| f(x+i y)\right|^{2} d x<\infty\right\} .
$$

As the Hardy-norm locally dominates the Bergman-norm on $T(\Omega)$, it follows hence $\mathcal{H}^{2}(T(\Omega))$ is complete, i.e. a Banach space. In fact, $\mathcal{H}^{2}(T(\Omega))$ is a Hilbert space as we will show in a moment. Then for $f \in \mathcal{H}^{2}(T(\Omega))$ and $y \in \Omega$ one has

$$
\int_{V}|f(x+i y)|^{2} d x=\int_{V^{*}}\left|\mathcal{F}\left(\left.f\right|_{V}\right)(\xi)\right|^{2} e^{-2\langle y, \xi\rangle} d \xi
$$

which is immediate from [21] Ch. III, §2. It follows that

$$
\|f\|_{\mathcal{H}}^{2}=\sup _{y \in \Omega} \int_{V^{*}}\left|\mathcal{F}\left(\left.f\right|_{V}\right)(\xi)\right|^{2} e^{-2\langle y, \xi\rangle} d \xi
$$

For $y \in V$ define $\cosh , \cosh _{y}: V^{*} \rightarrow \mathbb{C}$ by

$$
\cosh _{y}(\lambda)=\frac{1}{|W|} \sum_{s \in \mathcal{W}} e^{-2\langle y, s \lambda\rangle}=\frac{1}{|W|} \sum_{s \in \mathcal{W}} e^{-2\langle s y, \lambda\rangle}
$$

and

$$
\cosh (\lambda)=\cosh _{y_{o}}(\lambda)
$$

As with $\|\cdot\|_{L^{2}(V)}$ is $\mathcal{W}$-invariant and $\mathcal{F}$ is $\mathcal{W}$-equivariant, it follows from (17.6) that

$$
\|f\|^{2}=\sup _{y \in \Omega} \int_{V^{*}}\left|\mathcal{F}\left(\left.f\right|_{V}\right)(\xi)\right|^{2} \cosh (y, \xi) d \xi
$$


Now, for every $\lambda \in V$, the function $y \mapsto \cosh _{\mathbf{y}}(\lambda)$ is strictly convex on $\bar{\Omega}$; hence we have the inequality

$$
(\forall y \in \Omega)\left(\forall \lambda \in V^{*}\right) \quad \cosh _{y}(\lambda) \leq \cosh (\lambda) .
$$

and so it follows that

$$
\|f\|_{\mathcal{H}}^{2}=\int_{V^{*}}\left|\mathcal{F}\left(\left.f\right|_{V}\right)(\lambda)\right|^{2} \cosh (\lambda) d \lambda
$$

Define a $\mathcal{W}$-invariant measure $\mu_{0}$ on $V^{*}$ by

$$
d \mu_{0}(\lambda)=\cosh (\lambda) d \lambda \text {. }
$$

Theorem 7.8. The mapping

$$
\mathcal{H}^{2}(T(\Omega)) \rightarrow L^{2}\left(V^{*}, d \mu_{0}\right), \quad f \mapsto \mathcal{F}\left(\left.f\right|_{V}\right)
$$

is an isometric isomorphism. In particular, $\mathcal{H}^{2}(T(\Omega))$ is a Hilbert space.

A (continuous) multiplier on $V^{*}$ is a continuous map $m: \mathcal{W} \times V^{*} \rightarrow \mathbb{C}$ such that for all $s, w \in \mathcal{W}$ and $\lambda \in V^{*}$ we have

$$
m(s w, \lambda)=m(s, w \lambda) m(w, \lambda) .
$$

Assume from now on that $|m(s, \lambda)|=1$ for all $s \in \mathcal{W}$ and $\lambda \in V^{*}$. Then, because of the $\mathcal{W}$-invariance of $d \mu_{0}$, we can define a unitary representation of $\mathcal{W}$ on $L^{2}\left(V^{*}, d \mu_{0}\right)$ by

$$
\tau(s) f(\lambda)=m\left(s^{-1}, \lambda\right) f\left(s^{-1} \lambda\right) .
$$

As the Fourier transform is a unitary isomorphism, we have a unitary representation, also denoted by $\tau$, of $\mathcal{W}$ on $\mathcal{H}^{2}(T(\Omega))$ such that the Fourier transform is an intertwining operator. Denote the space of $\tau(\mathcal{W})$-invariant elements by the superscript $\tau(\mathcal{W})$. Then

Corollary 7.9. The Fourier transform is a unitary isomorphism

$$
\mathcal{F}: \mathcal{H}^{2}(T(\Omega))^{\tau(\mathcal{W})} \rightarrow L^{2}\left(V^{*}, d \mu_{0}\right)^{\tau(\mathcal{W})} .
$$

The Hardy space $\mathcal{H}^{2}(T(\Omega))^{\tau(\mathcal{W})}$ being a Hilbert space of holomorphic functions admits as such a reproducing kernel function $K(z, w)$ often called the Cauchy-Szegö-kernel. Notice that for fixed $w \in T(\Omega)$, the function $K_{w}(z)$ belongs to $\mathcal{H}^{2}(T(\Omega))^{\tau(\mathcal{W})}$ and that

$$
\left\langle f, K_{w}\right\rangle=f(w) \quad \text { for all } f \in \mathcal{H}^{2}(T(\Omega))^{\tau(\mathcal{W})} .
$$

Here of course $\langle\cdot, \cdot\rangle$ denotes the inner product on $\mathcal{H}^{2}(T(\Omega))^{\tau(\mathcal{W})}$.

We now determine $K(z, w)$ explicitly. For that define for $w \in V, \cos _{w}^{m}: V^{*} \rightarrow \mathbb{C}$ by

$$
\cos _{w}^{m}(\lambda):=\frac{1}{|\mathcal{W}|} \sum_{s \in \mathcal{W}} m(s, \lambda)^{-1} e^{i\langle w, s \lambda\rangle}
$$

and note that

$$
\frac{\cos _{w}^{m}}{\cosh } \in \mathcal{H}^{2}(T(\Omega))^{\tau(\mathcal{W})}
$$

Write $(\cdot \mid \cdot)$ for the inner product on $L^{2}\left(V^{*}, d \mu_{0}\right)^{\tau(\mathcal{W})}$. For $f \in \mathcal{H}^{2}(T(\Omega))^{\tau(\mathcal{W})}$ let $F=\mathcal{F}\left(\left.f\right|_{V}\right)$. 
It follows from Corollary 7.9 that $\left\langle f, K_{w}\right\rangle=\left(F \mid \mathcal{F}\left(\left.K_{w}\right|_{V}\right)\right)$. On the other hand we have

$$
\begin{aligned}
f(w) & =\int_{V^{*}} F(\lambda) e^{-i\langle w, \lambda\rangle} d \lambda \\
& =\int_{V^{*}} F(\lambda) \frac{e^{-i\langle w, \lambda\rangle}}{\cosh (\lambda)} d \mu_{0}(\lambda) \\
& =\int_{V^{*}} m\left(s^{-1}, \lambda\right) F\left(s^{-1} \lambda\right) \frac{e^{-i\langle w, \lambda\rangle}}{\cosh (\lambda)} d \mu_{0}(\lambda) \\
& =\int_{V^{*}} F(\lambda) m\left(s^{-1}, s \lambda\right) \frac{e^{-i\langle w, s \lambda\rangle}}{\cosh (\lambda)} d \mu_{0}(\lambda) \\
& =\int_{V^{*}} F(\lambda) \frac{\cos _{w}^{m}(\lambda)}{\cosh (\lambda)} d \mu_{0}(\lambda)
\end{aligned}
$$

and thus

$$
\mathcal{F}\left(\left.K_{w}\right|_{V}\right)(\lambda)=\frac{\cos _{w}^{m}(\lambda)}{\cosh (\lambda)}
$$

Theorem 7.10. The reproducing kernel for the Hardy space $\mathcal{H}^{2}(T(\Omega))^{\tau(\mathcal{W})}$ is given by

$$
\begin{aligned}
K(z, w) & =\int_{V^{*}} \frac{\left(\frac{1}{|\mathcal{W}|} \sum_{s \in \mathcal{W}} \frac{e^{i\langle s(z), \lambda\rangle}}{m(s, \lambda)}\right) \cdot\left(\overline{\left.\frac{1}{|\mathcal{W}|} \sum_{s \in \mathcal{W}} \frac{e^{i\langle s(w), \lambda\rangle}}{m(s, \lambda)}\right)}\right.}{\cosh (\lambda)} d \xi \\
& =\int_{V^{*}} \frac{\cos _{z}^{m}(\lambda)}{\cosh (\lambda)} \cos _{-w}^{m}(\lambda) d \lambda .
\end{aligned}
$$

Example 7.11. The equation (7.10) can be evaluated in the relevant special cases. Let us for example consider the case of $V=\mathbb{R}, \Omega=]-1,1[, \mathcal{W}=\mathrm{O}(\mathbb{R}) \simeq\{\mathbf{1},-\mathbf{1}\}$ and $m(s, \lambda)=1$. Using the standard measure on $\mathbb{R}$ we get the following from (17.10) and [2, Sect. 1.9, formula (12):

$$
\begin{aligned}
K(z, w) & =\frac{1}{\sqrt{2 \pi}} \int_{\mathbb{R}} \frac{\cos (z \xi) \cos (\bar{w} \xi)}{\cosh (2 \xi)} d \xi \\
& =\sqrt{\frac{\pi}{2}} \frac{\cosh \left(\frac{\pi}{4} z\right) \cdot \cosh \left(\frac{\pi}{4} \bar{w}\right)}{\cosh \left(\frac{\pi}{2} z\right) \cdot \cosh \left(\frac{\pi}{2} \bar{w}\right)} .
\end{aligned}
$$

\section{REFERENCES}

[1] J. Arthur, A theorem on the Schwartz space of a reductive Lie group, Proc. Natl. Acad. Sci. USA 72, 4718-4719 (1975)

[2] A. Erdelyi et al., Tables of Integral Transforms, Volume I, McGraw-Hill, 1954.

[3] Bernstein, J., Analytic continuation of generalized functions with respect to a parameter, Funkcional. Anal. i Priložen. 6 (1972), no. 4, 26-40.

[4] P. Delorme and M. Flensted-Jensen, Towards a Paley-Wiener theorem for semisimple symmetric spaces, Acta Math. 167 (1991), 127-151. 
[5] J. Faraut, Formule de Gutzmer pour la complexification d'un espace Riemannien suymétrique, Rend. Mat. Acc. Lincei s. 9 , v. 13:233-241 (2002).

[6] Gindikin, S., SO(1;n)-twistors, J. Geom. Phys. 26 (1998), no. 1-2, 26-36.

[7] Gindikin, S., Integral geometry on SL(2; R), Math. Res. Lett. 7 (2000), no. 4, 417-432.

[8] S. Gindikin and B. Krötz, Invariant Stein Domain in Stein Symmetric Spaces and a Nonlinear Complex Convexity Theorem, IMRN 18 (2002), 959-971.

[9] S. Gindikin, B. Krötz and G. Ólafsson, Holomorphic $H$-spherical distribution vectors in principal series representations, Invent. Math. 158 (2004), 643-682.

[10] _ Horospherical model for the holomorphic discrete series and the horospherical Cauchy transform. math.RT/0411564, to appear in Compositio Math.

[11] Harish-Chandra, Spherical Functions on a Semisimple Lie Group I. Amer. J. Math. 80 (1958), 241-310.

[12] Harish-Chandra, Discrete Series for Semisimple Lie Groups II, Acta Math. 116 (1966), 1-111.

[13] S. Helgason, Geometric Analysis on Symmetric Spaces, Math. Surveys and Monographs 39, Amer. Math. Soc., 1994.

[14] J. Hilgert and G. Ólafsson, Causal Symmetric Spaces, Geometry and Harmonic Analysis. Perspectives in Mathematics 18, Academic Press, 1997.

[15] B. Krötz, G. Ólafsson and R. Stanton, The image of the heat kernel transform on Riemannian symmetric spaces of the noncompact type, IMRN 22 (2005), 1307-1329.

[16] B. Krötz and M. Otto, A Refinement of the Complex Convexity Theorem via Symplectic Techniques, Proc. Amer. Math. Soc. 134 (2) (2006), 549-558.

[17] T. Matsuki, The orbits of affine symmetric spaces under the action of minimal parabolic subgroups, J. Math. Soc. Jpn. 31 (1979), 331-357.

[18] G. Ólafsson, Fourier and Poisson transformation associated to a semisimple symmetric space, Invent. Math. 90 (1987) 605-629.

[19] G. Ólafsson and H. Schlichtkrull, The Segal-Bargmann transform for the heat equation associated with root systems, submitted, math.AP/0509057.

[20] T. Oshima and J. Sekiguchi, Eigenspaces of invariant differential operators on an affine symmetric space, Invent. Math. 57 (1980), no. 1, 1-81.

[21] E. Stein and G. Weiss, Introduction to Fourier Analysis on Euclidean Spaces, Princeton University Press, 1971.

Department of Mathematics, Rutgers University, New Brunswick, NJ 08903

E-mail address: gindikin@math.rutgers.edu

Max-Planck-Institut für Mathematik, Vivatsgasse 7, D-53111 Bonn, Germany

E-mail address: kroetz@mpim-bonn.mpg.de

Department of Mathematics, Louisiana State University, Baton Rouge, LA 70803, USA

E-mail address: olafsson@math.lsu.edu 